\title{
Trade Mark Cluttering: An Exploratory Report
}

Research commissioned by the Intellectual Property Office, and carried out by: Georg von Graevenitz*, Christine Greenhalgh†, Christian Helmersł, Philipp Schautschick§ April 2012 
* GEORG VON GRAEVENITZ (g.graevenitz@uea.ac.uk) works as Senior Lecturer in Innovation Management at University of East Anglia's London campus. His research interests are on the management and effects of intellectual property rights, the management of innovation and entrepreneurship. He has consulted on intellectual property rights for the European Commission and UK IPO and has published his research on intellectual property and entrepreneurship in international journals such as Economics Letters and the Journal of Economic Behavior and Organization.

†CHRISTINE GREENHALGH (christine.greenhalgh@ economics.ox.ac.uk) is Professor of Applied Economics, Emeritus, University of Oxford and Emeritus Fellow of St Peter's College. Her research Interests include intellectual property and innovation, industrial and firm performance, technical and structural change, and vocational training. Her textbook (co-author Prof. Mark Rogers) - Innovation, Intellectual Property and Economic Growth - was published by Princeton University Press in 2010.

${ }^{\ddagger}$ CHRISTIAN HELMERS (christian.helmers@uc3m.es) is Assistant Professor of Management at Universidad Carlos III de Madrid, Spain. He is also a Research Affiliate with the Spatial Economics Research Centre at LSE and the Centre for the Study of African Economies at Oxford University. Christian has been a research economist at LSE as well as a visiting researcher at UC Berkeley and LMU Munich. He currently is a visiting research scholar at Stanford University.

§PHILIPP SCHAUTSCHICK (philipp.schautschick@gmail. com) is a doctoral candidate at the International Max Planck Research School for Competition \& Innovation and the Ludwig Maximilians University, Munich. He holds an MPhil in economics from Oxford University. His research focuses on the economic theory of intellectual property (in particular on patents and trade marks) and the impact of intellectual property on competition.

\section{ISBN: 978-1-908908-29-2}

Trade Mark Cluttering: An Exploratory Report

Published by The Intellectual Property Office 13th April 2012

\section{0 \\ (C) Crown Copyright 2012}

You may re-use this information (excluding logos) free of charge in any format or medium, under the terms of the Open Government Licence. To view this licence, visit http://www.nationalarchives.gov. uk/doc/open-government-licence/

or email: psi@nationalarchives.gsi.gov.uk

Where we have identified any third party copyright information you will need to obtain permission from the copyright holders concerned.

Any enquiries regarding this publication should be sent to:

The Intellectual Property Office

Concept House

Cardiff Road

Newport

NP10 8QQ

Tel: 03003002000

Minicom: 03000200015

Fax: 01633817777

e-mail: information@ipo.gov.uk

This publication is available from our website at www.ipo.gov.uk 


\section{CONTENTS}

Abstract 3

1. Introduction

2. Part I - Conceptual Analysis 7

$\begin{array}{lll}2.1 & \text { Trade Mark Registers and Cluttering } & 7\end{array}$

2.1.1 Patent Thickets 8

2.1.2 Trade Marks 9

$\begin{array}{lll}2.1 .3 & \text { Trade Mark Cluttering }\end{array}$

$\begin{array}{lll}2.2 & \text { Literature } & 14\end{array}$

$\begin{array}{lll}2.3 & \text { Background: UK and OHIM Marks } & 16\end{array}$

3. Part II - Empirical Analysis 18

$\begin{array}{lll}3.1 & \text { Analysis of UK Data } & 18\end{array}$

$\begin{array}{lll}\text { 3.1.1 UK Data - OFLIP } & 18\end{array}$

$\begin{array}{lll}3.1 .2 & \text { Descriptive Analysis } & 18\end{array}$

3.1.3 Growth Regression 24

$\begin{array}{lll}3.1 .4 & \text { Summary } & 26\end{array}$

$\begin{array}{lll}3.2 & \text { Analysis of OHIM Data } & 27\end{array}$

$\begin{array}{lll}\text { 3.2.1 } & \text { Trade marks at OHIM } & 29\end{array}$

3.2.2 The Breadth of Trade Mark Applications 31

3.2.3 A Policy Shock: Abolishing Refusals based on Relative
Grounds Examination

3.2.4 Many Simultaneous Applications 42

4. Conclusion 46

References $\quad 48$

Appendix 51

Nice classification $\quad 51$ 



\section{Abstract}

This report explores the problem of "cluttering" of trade mark registers. The report consists of two parts: the first presents a conceptual discussion of "cluttering" of trade mark registers. The second part provides an exploratory empirical analysis of trade mark applications at the UK Intellectual Property Office (IPO) and the European trade mark office (OHIM). This part contains results of a descriptive and an econometric analysis.

According to our definition, cluttering arises where firms hold trade marks that are overly broad or unused raising search costs for later applicants. The report distinguishes between three mechanisms that can lead to cluttering. It also considers a series of mechanisms that work against cluttering. This discussion is based on a review of the previous literature.

The tentative empirical evidence provided in the second part of the report suggests that trade marks are more frequently registered in several classes at the same time and also that firms in pharmaceuticals increasingly resort to multiple simultaneous applications to ensure that they will register at least one trade mark. There is also some evidence that firms seek to avoid mechanisms such as relative grounds examination which can prevent cluttering. Finally, we report direct survey-based evidence that applicants perceive cluttering to be a problem in specific fields and countries.

However, our exploratory analysis does not provide strong evidence that cluttering has already become a systemic problem for the trade mark systems that is comparable to the effect of patent thickets for patent systems.

Keywords: Trade marks, Cluttering 



\section{Introduction}

There has been increasing concern over the emergence of so-called patent thickets due to sharp increases in overall patent filings, in particular in certain sectors such as computer and telecommunications (see Chapter 6 in the Hargreaves Review (Hargreaves, 2011); von Graevenitz et al. (2007); National Research Council (2004); Federal Trade Commission (2003)). Very recently this has led to reform of the US patent system as well as more limited administrative changes within the patent system administered by the European Patent Office (EPO). The growing evidence for problems arising from patent thickets, motivates us to ask whether similar problems stemming from excessive applications for intellectual property rights arise for other forms of intellectual property, notably trade marks in form of "cluttering".

The aim of this report is to clarify the concept of trade mark cluttering and to provide as much evidence regarding the phenomenon as we could obtain from existing data sources. ${ }^{1}$ Accordingly the report consists of two parts: the first contains a literature review and a definition of cluttering and mechanisms contributing to it. In the second part we provide initial indicators of where clutter exists (or not).

For the purposes of this study we define cluttered trade mark registers as registers containing such a large number of unused or overly broad trade marks ${ }^{2}$, that the costs of creating and registering new marks substantially increase for other applicants. Furthermore, the problem of cluttering becomes systemic if existing levels of cluttering lead later applicants to adopt application strategies that further contribute to the problem.

The proper functioning of trade mark registers is important for several reasons: first; trade marks are ubiquitous and facilitate many modern forms of commerce such as product differentiation, franchising and brand extension (Griffiths, 2007, 2009); second, there is strong evidence that many firms rely on trade marks when appropriating benefits not just from product differentiation but also from innovation. ${ }^{3}$ As a consequence demand for trade marks is high and growing around the world. ${ }^{4}$ Figure 1 below demonstrates this very impressively for the United States.

The figure shows that demand for patents and trade marks has been running in parallel at United States Patent and Trade Mark Office (USPTO) and that this demand started to accelerate substantially after 1984. In the case of patents, the steep increase in demand has created negative externalities for applicants. Not only are there enormous backlogs of unexamined patents numbering in the hundreds of thousands ${ }^{5}$ which creates uncertainty and leads to

1 For this purpose, we draw on a firm-level database for the UK that contains marks registered with the UK Intellectual Property Office (IPO) and the European trade mark office (OHIM). We also make use of data on trade mark applications by other nationals at OHIM.

2 Overly broad in this context means seeking protection in more classes than are required.

3 The available survey evidence from the different rounds of the Community Innovation Survey (CIS) for the UK indicates that firms favour trade marks to appropriate returns to innovation over all other forms of registered intellectual property (see Hall et al. (2011)). Other forms of IP included in the CIS are patents and registered designs.

4 According to the Facts \& Figures 2009-2010, the IPO received a total of 15,194 patent and 61,318 trade mark filings from UK residents in 2010.

5683,991 at USPTO and 490,000 (2010) at EPO. 
strategic behaviour (von Graevenitz et al., 2007). Additionally, the generally low quality of patents (Lemley and Shapiro, 2005; Lei and Wright, 2009) and a high degree of overlap between them lead to litigation, hold-up through injunctions and mergers and acquisitions that are motivated by the desire to protect against patent litigation (e.g. Google-Motorola).

\section{Figure 1: Demand for Patents and Trade Marks at USPTO}

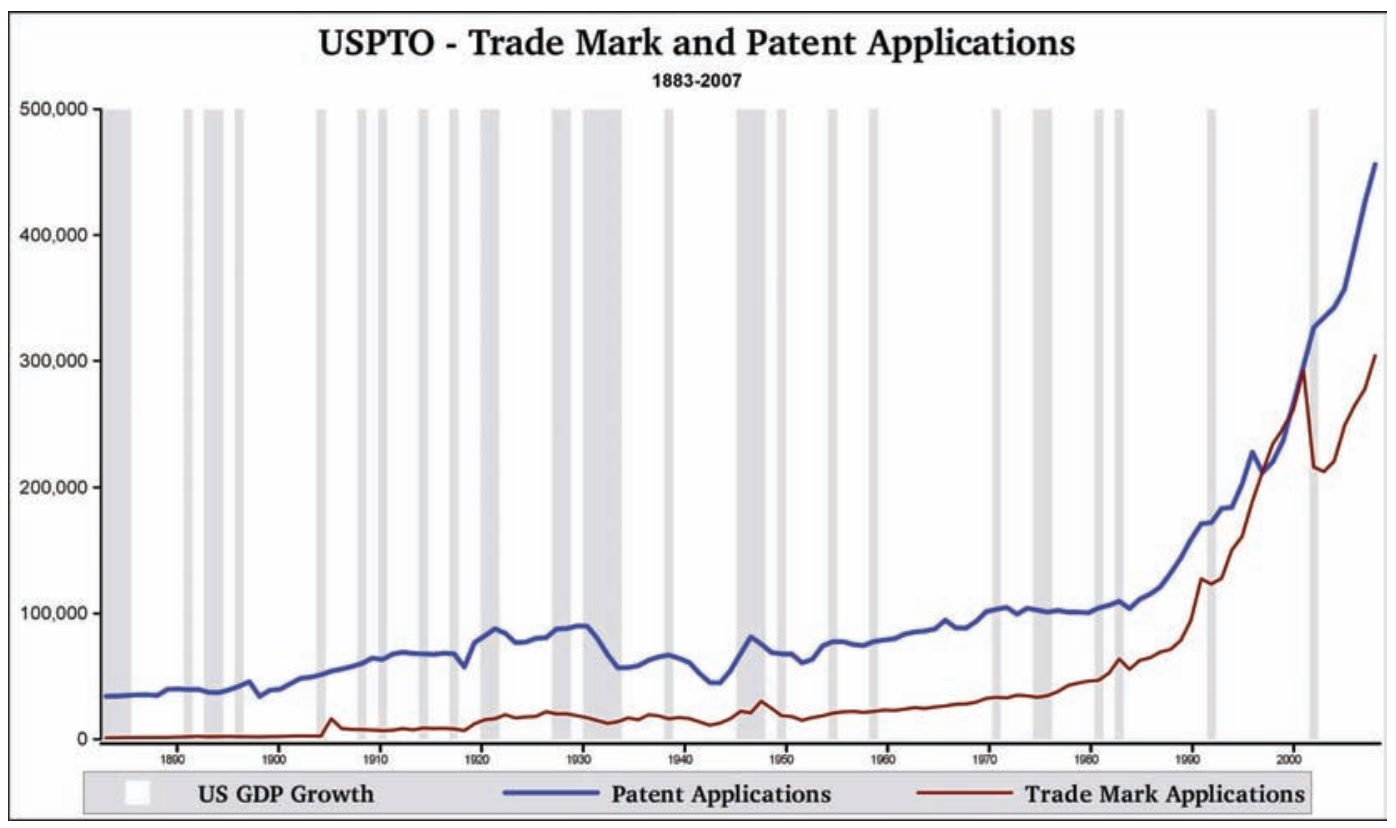

Note: This graph depicts periods of negative (grey) and positive (white) US GDP growth in the background. These are based on data obtained from the Federal Reserve Bank of St. Louis. The graph provides evidence of growth of demand for trade marks and patents at USPTO. Data for these series was collected from World Intellectual Property Office (WIPO).

Trade marks differ from patents in that they are not based on claims on an underlying technology that may overlap. Rather the mark is based on a sign or word registered in one or more classes that delimit areas of economic activity. Thus trade marks are arguably somewhat simpler property rights and usually the degree of protection they confer is more clearly delineated than in the case of patents. This means that offices can clear them quickly and there is less scope for strategic behaviour during application.

Nevertheless, as we will discuss in more detail below, the increasing volume of registered trade marks affects businesses by increasing the costs of using and registering further new marks. This is not because administrative or agents' fees have risen for each individual trade mark. ${ }^{6}$ Rather, higher costs result from increased effort required to search an ever growing registry of existing marks, increased difficulty in finding new, distinctive yet compelling marks and an increased likelihood of facing opposition, or even litigation at the crucial stage of market introduction of new products or services. Anecdotal evidence suggests that these costs are substantial. 
As we show below it is highly likely that one consequence of the strong increase in demand for trade marks has been that registers around the world harbour trade marks that are not in use and are unlikely to be put into use. Due to the lack of very effective mechanisms which would quickly remove such trade marks "cluttering" of trade mark registers could turn into a growing problem. As with patents this would be due to feedback effects. As unused trade marks become more prevalent, the cost of identifying new names or signs rises and the probability of successfully registering new marks falls. Some strategies that firms can adopt to circumvent these problems will then make the problem bigger. For instance, firms may seek to apply for more than one mark simultaneously to raise the likelihood of registering at least one mark. Also firms may seek to register their marks in more classes than strictly required by their current business needs to ensure that the extension into new markets is not blocked by others' trade marks registered at a later date.

As noted above the report consists of two parts. The first part is conceptual whereas the second is empirical. The conceptual first part consists of Sections 2.1-2.3. Section 2.1 provides an indepth discussion of the notion of trade mark cluttering. We review prior literature on cluttering in Section 2.2. Section 2.3 provides details on the UK and OHIM trade mark systems that we analyse. The second, empirical part of the report consists of Sections 3.1 and 3.2. These provide an exploratory empirical analysis to determine whether there are indications that trade mark cluttering may affect applicants in the UK (Section 3.1) and in Europe (Section 3.2). Section 4 concludes the report, summarising our findings.

\section{Part I - Conceptual Analysis}

\subsection{Trade Mark Registers and Cluttering}

Both trade marks and patents are generally registered with an office that administers the process of examination and registration of these property rights. While patents and trade marks have been registered in several jurisdictions for well over a century the demand for these IP rights has risen dramatically relative to historical demand since the mid 1980's. For example, the register at OHIM has grown since it opened for business in 1996 from 43,144 to over 760,000 registered trade marks with 100,795 marks added just in $2010 .{ }^{7}$ Similarly the registers at DPMA (Deutsches Patent- und Markenamt), IPO and USPTO hold a further 778,000 (2009), 474,245 (2009) and 1,684,949 registered trade marks, respectively.

In this section we define cluttering of trade mark registers and review mechanisms that could lead to cluttering and others that may work against it. First, we briefly review the literature on patent thickets to provide a comparison for our discussion of trade mark cluttering. We do this as the notion of patent thickets and the problems associated with them are firmly established in the economics literature, whereas the problem of trade mark cluttering is discussed only by legal scholars to date. Next, we describe the legal mechanisms underlying trade mark registration

$7 \quad$ Figures from OHIM statistical reports. 
and we discuss the role of trade mark protection in the economy. Then, we turn to the definition of cluttering and mechanisms that support or counteract cluttering.

\subsubsection{Patent Thickets}

According to Shapiro (2001), patent thickets are sets of overlapping patents with uncertain validity and scope. The existence of patents with overlapping claims creates a web of interlocking property rights that firms have to disentangle if they seek to use a technology affected by a patent thicket without risking costly infringement suits.

Patent thickets arise because in many technological fields technical inventions are combined in a modular fashion within a final product. Often substitute solutions exist for any particular component or function within such a product and each component is protected by a patent. Complex products can consist of hundreds or even thousands of such patent protected components. Also, each individual component may be suitable for inclusion in more than one product. This creates a very complex web of technological dependencies between technological components (Grindley and Teece, 1997; Hall and Ziedonis, 2001) ${ }^{8}$.

The property rights (patents) to components included in products such as a mobile phone will often be dispersed among a large number of companies. For any one company seeking to market such a complex product the main challenge is to ensure that all firms holding property rights to components have agreed to license their components or rights at acceptable cost.

Frequently firms that are not party to the agreements covering successful complex products seek to obtain a share of the profits from sales of such products by bringing a claim of infringement of patents against the firm marketing the product. Where such actions lead to injunctions against the producer of a complex product, this can be very costly and damaging for this firm. As a result firms have pursued strategies that insure them against such patent suits and injunctions. These strategies are otherwise unproductive and add substantially to the costs of doing business in some industries. Therefore, some commentators assert that patent systems world wide are "broken" (Jaffe and Lerner, 2004; Bessen and Meurer, 2008). An important aspect of being broken is the scope for strategic gaming of the application process and the strategic use of injunctions and other legal mechanisms to gain competitive advantage. These problems are particularly virulent in the United States but also affect patent applications at the European Patent Office (von Graevenitz et al., 2007).

Patent thickets are more likely to arise if patents are poorly delineated. Also patent thickets will be more likely to arise if patents that are simply invalid are granted because examiners do not discover existing relevant prior art. Both these problems arise more frequently if patent examiners are poorly incentivised to refuse badly worded or marginal patent applications as has been the case in the US (Lei and Wright, 2009). Finally, patent thickets will matter more if the courts are more willing to issue injunctions against firms that may be infringing on a patent.

8 It is important to note that patent thickets are not just the result of a large volume of patent applications or grants. The modular interdependence of technology is an important part of the definition of what a patent thicket is Shapiro (2001); lyama (2005). This is the main reason for the overlap of patent rights and the ability of nonpracticing entities to hold up producing firms. 


\subsubsection{Trade Marks}

Registered trade marks have the objective to protect a unique sign, mark or symbol in order to make a product or service distinguishable from competing products. ${ }^{9}$ The trade mark can be any word(s), graphics, figures, images, a combination of these or similar that act as a distinguishing feature. Trade marks can also be distinctive shapes, colours or sounds. Hence, a trade mark protects characteristics that give products and services a recognisable feature which may be used by customers to identify the origin of the product thereby also conveying further information such as a reputation of the manufacturer to customers.

From a legal point of view, the registration of a trade mark allows its owner to (i) advertise a sign as a registered trade mark, (ii) stop rivals imitating ('passing-off') the products of others, (iii) license the trade mark for use by third parties, and (iv) sell the trade mark to others. ${ }^{10} \mathrm{~A}$ trade mark application has to pass a number of tests, the principle being that the sign should be distinctive. Signs may also be prohibited where previous and conflicting rights exist. In addition, in the US and in most EU jurisdictions, a trade mark is required to be used. However, the use requirement is treated differently in these jurisdictions which has important implications as we discuss below. Most importantly, it is much more stringently enforced in the US than in Europe.

Trade marks are a much more widespread form of IP than patents. In the UK, for example, Rogers et al. (2009) have found that firms register more than twice as many trade marks as patents each year. Moreover, at least in the UK, there has been a strong increase in registered trade marks. There is little dispute among economists with regard to private as well as social benefits accruing from trade marks in general. As stated by Boldrin and Levine (2008) there are strong economic advantages in allowing market participants to voluntarily identify themselves (2008: 7), i.e., there are some sound economic reasons for allowing firms to register trade marks. Notwithstanding, there is relatively little research actually quantifying the positive effects of trade marks on firm performance and effectively none on potential negative effects of trade mark cluttering. ${ }^{11}$ Greenhalgh et al. (2011) provide a first empirical analysis to firms' incentives to apply for trade marks.

Trade marks, therefore, allow producers to set their products and services apart from those of competitors and to inform customers on unobservable product characteristics. This allows consumers to identify goods and services by a given producer and hence to mitigate the inherent information asymmetry between buyers and sellers. According to Landes and Posner (1987), trade marks therefore fulfil mainly two roles: first, trade marks reduce consumer search costs; second, trade marks provide incentives for firms to invest and maintain a certain quality of their goods and services. Landes and Posner (1987) note in particular the self-enforcing mechanism provided by trade marks: firms will seek a trade mark if they are able to invest in quality and given that they are able to invest in quality, a trade mark lowers consumers' search costs. The main economic justifications for the use of trade marks is thus that they can help to solve the information asymmetry between seller and buyer.

9 There are two other forms of registered marks: certification marks and collective marks. But we do not discuss these registered marks here as they are not part of our analysis.

10 E.g. Council Regulation (EC) No. 40/94 (1994) Article 17.

11 For a general overview of the economics of trade marks see Landes and Posner (1987) and Economides (1988); for an overview of how trade marking affects firm performance in the UK see Helmers and Rogers (2009). 
By allowing the producer to protect their reputation trade marks effectively allow firms to build brands. To do this firms advertise and simultaneously invest in product quality (Cabral, 2000; Sutton, 2007). The connection between the brand image and the quality of the product as perceived and experienced by the consumer gives firms strong incentives to improve and maintain product quality (Rob and Fishman, 2005). The brand reputation associated with well known products makes entry into markets protected by such reputation more difficult. These "entry barriers" are an important incentive for the creation of reputation and therefore are not in and of themselves problematic, in spite of the fact that barriers to entry are often regarded as barriers to competition.

\subsubsection{Trade Mark Cluttering}

The aim of this section is to delineate circumstances in which trade mark cluttering could arise and to describe mechanisms underlying the phenomenon. We also discuss mechanisms that might reduce the threat of cluttering or allow firms to circumvent the problem. As noted above we define cluttered trade mark registers as registers containing such a large number of unused or overly broad trade marks 12 , that the costs of creating and registering new marks substantially increase for other applicants. Furthermore, the problem of cluttering becomes systemic if existing levels of cluttering lead later applicants to adopt application strategies that further contribute to the problem.

This definition suggests that where cluttering arises it is mainly a problem of absence of housekeeping mechanisms; trade mark offices that cannot remove unused trade marks from their register provide incentives for the creation of more unused marks ${ }^{13}$ This feedback mechanism mimics that underlying the growth of patent thickets: large numbers of potentially threatening low quality patents create incentives for more low quality patent applications, created to defend against injunction. The main question to be answered in future work on cluttering is one of cost: how important is the economic cost of cluttering relative to the costs of cleansing the register.

Landes and Posner (1987) assert that the space of names is infinite and therefore competition for individual trade mark names is not likely to arise. In particular, they state that prior to establishing a trade mark, the distinctive yet pronounceable combinations of letters to form words that will serve as a suitable trade mark are as a practical matter infinite, implying a high degree of substitutability (1987: 274). Nevertheless, Landes and Posner (1987) distinguish between three types of marks, which differ in terms of their degree of substitutability. The availability of so-called fanciful marks, that is marks consisting of invented words, such as "Exxon" are in principle infinite. Suggestive marks, that is marks indicating the use of the protected product, e.g. "The Financial Times", display a lower degree of substitutability. According to Landes and Posner (1987), the potentially most limited supply of marks applies to so-called descriptive marks. Such marks describe the product/service they apply to, such as "Holiday Inn" for a hotel chain. Provided that a given product has only so many attributes that interest buyers (1987: 290), cluttering of such marks may make it more costly for entrants to come up with names without relying on existing descriptive marks. Similar to such descriptive marks are marks that consist of common symbols and single colours. These marks are less distinctive and more limited in supply. Hence, a large number of such marks may make it more

12 Trade marks that are registered in a class in which their owners currently have no business, so that the mark could not be enforced against another on the basis of the registration in that class.

13 Section 3.2.2 provides evidence for such a feedback mechanism in the area of pharmaceuticals. 
difficult for entrants to find distinctive marks. Therefore, despite the general view that trade marks are in principle unlimited in supply, Landes and Posner (1987) recognise the potential for some scarcity of trade marks to arise.

There are two implications that lead from scarcity of potential names to increased costs of trade mark protection:

i) We might argue that while it is true that there are infinitely many possible words that could be used as marks, the space of marks that have desirable connotations while being sufficiently distinct from existing trade marks is not necessarily also infinite. Certainly, the identification of such marks is quite a costly process involving highly paid specialists.

ii) More fundamentally, we could argue that as with patents the problem is not in competition for individual rights but in the interdependencies between the property rights. Such interdependencies may mean that a single registered trade mark rules out a large number of similar marks. Below we consider several mechanisms giving rise to such interdependencies.

Thus scarcity of names would certainly suggest that trade marks that are unused should not "clutter" trade mark registers because they might prevent more profitable uses of the same name or similar names. However, even in the absence of scarcity cluttering can matter if trade mark registers become large. The larger a register, the bigger the problem of potential infringement for new applicants. In the case of the European trade mark system it is already widely acknowledged that an exhaustive search of all registers containing trade marks that might be the basis of opposition is prohibitively costly (Kur et al., 2011). When new products or services are launched on a market the delay created by opposition can be extremely costly for firms.

One way to ensure timely registration of a trade mark in the face of a large register is to apply for more than one trade mark at the same time. Simultaneous applications give rise to an important problem - only one of the marks will be used, the remainder will clutter the register for some time, depending on the severity of use requirements. This makes the register even larger, creating a feedback loop with potential for ever increasing amounts of unused marks.

The core problem of cluttering is that of the creation and existence of unused trade marks on trade mark registers that might block entry of new products. What complicates the analysis of such marks is the fact that trade marks may also become unused because businesses fail. In this case a mark will have been in use for some time and then fall into disuse. We are more concerned with marks that never enter into use and for which it is clear soon after registration that this is likely to be the case. This includes also unused marks that are held for strategic reasons by companies.

The empirical question that we seek to provide a very provisional answer to is this: is cluttering important enough to raise firms' costs of doing business substantially? ${ }^{14}$

14 It may be useful to note at this point that Section 3.2.1 contains direct evidence from a survey of users on this question. This reflects the opinions of owners of OHIM trade marks and trade mark representatives (agents) at OHIM. 
Our discussions with examiners at IPO, representatives of firms and business associations reveal the following three firm strategies which can contribute to cluttering of a trade mark register:

- Firms may be applying for protection in more Nice classes than strictly needed. This means that the trade mark register is not as good a reflection of the use of trade marks in the course of trade as it should be.

We investigate this by analysing the breadth of UK marks in terms of number of Nice classes in Section 2.3 below. Additionally, we investigate OHIM's 3 for 1 policy in the pricing of applications in Section 3.2.2 below.

- In some fields the interdependencies between trade marks are much stronger than envisaged by Landes and Posner (1987). For instance, in the field of pharmaceuticals, medical regulators increasingly impose the standard that doctors and pharmacists should not be able to confuse trade marked pharmaceutical products if a spelling error or messy handwriting makes the name ambiguous. This raises the bar for applicants substantially; not only must they ensure registration of the trade mark, but also confirmation of the mark by the regulator. It can be shown that firms respond to this by simultaneously applying for more than one trade mark

In Section 3.2.4 we analyse this case briefly.

A similar problem may arise where firms with international reach are attempting to create a trade mark with global reach. This requires that the mark not be rejected in any of a whole series of jurisdictions. The greater the number of jurisdictions the more likely it is that the firm will need to simultaneously apply for more than one trade mark to ensure that at least one mark will be registered in all jurisdictions simultaneously.

We are not yet in a position to investigate this case. The necessary data work to link multiple registers is currently ongoing.

- A slightly different, but similar case emerges where firms apply for marks that they do not have an immediate use for but that might be used in the future. Firms may seek to protect such marks if they anticipate that an extension of their product range is likely in order to prevent others from encroaching on an otherwise logical extension of their naming scheme. Again these marks will contribute to cluttering of trade mark registers. Examples here are some of the letter combinations protected by Daimler Benz: e.g. $U$ 200. Another example is given by BMW who have maintained the mark "8 Series" although the last product marketed under that name was manufactured in 1989.

- There is some anecdotal evidence that large firms use defensive trade mark applications by registering many marks which are not being used but which are close to their major mark, in order to ring fence the field. We have not as yet been able to investigate the significance of this phenomenon.

All of the above mechanisms explain why firms may have incentives to apply for more trade mark space than they actually use at any given time. 
A number of incentives work against this tendency for excessively broad applications. Trade mark offices levy fees for applications by the number of classes that firms apply for. Typically these fees increase linearly in the number of classes ${ }^{15}$. Furthermore trade mark systems incorporate a number of mechanisms that can prevent or reduce cluttering:

Renewal - Trade marks are registered for 10 years at a time in most trade mark registers world wide. Firms may renew registered marks by paying a fee. No further examination of the marks use is undertaken. In Section 3.2.2 we briefly discuss evidence that renewal helps to reduce cluttering as unused trade marks are abandoned. This mechanism is not very effective as unused trade marks will affect many other firms' costs of search within a decade.

Cancellation - At OHIM and national trade mark offices registrations can be cancelled for a number of reasons. For example, trade marks may be revoked if they are not used at any point within the five years following registration. Trade marks may also be cancelled (declared invalid) if they were, in fact, generic at the time of application (and have not acquired a distinctive character since) or where an earlier, conflicting right existed at time of application. Cancellations are currently very rare although in principle they offer an important mechanism for firms to clear the register of unused marks. Apparently, it is currently more cost effective to develop a new trade mark name than to invest in the cancellation of existing names that may be unused in order to facilitate registration of a new mark.

Opposition - Opposition at both OHIM and IPO allows owners of existing trade marks to bar registration of applications that they perceive to be too similar to their own marks. At OHIM the probability of opposition is comparatively high. It would seem that this mechanism does provide a bar to cluttering. However, opposition also provides an important incentive for applicants to clutter: in order to ensure registration in the face of opposition applicants can resort to multiple simultaneous applications, as they do in pharmaceuticals. Also, opposition can only be effective where the applicant's trade mark is too similar to existing trade marks.

Use requirement - As noted above trade marks can be challenged if they are unused. At USPTO this requirement is enforced very strictly. At OHIM and IPO the five year grace period allows firms to hold unused trade marks on the register for a long period. It also allows these firms to oppose later applications without proof of use if the earlier mark is not yet five years old at the commencement of opposition procedures. The use requirement is closely connected to both opposition and cancellation procedures. It acts as a complement to both. Strict use requirements can make cancellation and opposition more effective in reducing cluttering and vice versa.

Having considered these mechanisms that are bound to the rules and regulations governing trade mark systems we turn next to private contracts. Where cluttering imposes an externality on an applicant we might expect this to be resolved by contract rather than through the more adversarial mechanisms reviewed above.

Licensing - Trade mark applicants facing the problem of cluttering might be able to surmount the problem by contracting if there are only very few owners of the marks causing a problem for the applicant. In this case a bilateral contract setting out the terms on which the two parties 
marks may coexist within a trade mark system can be drawn up. Such contracts are routinely made by German trade mark owners. How widespread the practice is in other jurisdictions is not documented or known to us. As an economist one is sometimes struck by the potential for "coordination" that such contracts might offer, however in most cases the contracts will arise between parties whose trade marks are similar but whose underlying products are not.

Transfer - This is a variant of the above case. The owner of an unused, not needed trade mark might simply agree to sell it to a later applicant. We do not know of any quantitative analysis of how often marks are transferred between firms nor of the reasons giving rise to such transfers.

At present we are not in a position to evaluate how well such mechanisms regulate the level of applications for unused trade marks. However, Section 3.2.3 provides a first analysis of the effects of removing the refusal of trade marks on the basis of a relative grounds examination (RGE) at IPO. This procedure allowed the trade mark office to determine whether trade mark applications were too similar to registered trade marks. RGE might have reduced the flow of surplus marks somewhat and we analyse its effects on the demand for new marks.

\subsection{Literature}

This section offers a brief review of the existing academic literature on trade mark cluttering. In fact, the economics and management literature is silent on the issue. Legal scholars, in contrast, are aware of the potential of a cluttered register, i.e., a trade mark register full of unused trade marks. Their main concern is that firms will find it harder to clear new names for their marks. However, it is hardly mentioned that this could be an outcome of strategic behaviour, i.e., that firms clutter the register in order to raise rivals costs or seek broad protection to create barriers to entry.

Looking beyond the European Union, one finds that the discussion in the US about the userequirement versus a registration system without ex parte examination frequently mentions the issue of trade mark register cluttering. Winner (1982) points out that countries exercising a trade mark system without use requirement may be 'pirate-countries', as shady individuals could register trade marks well known in one country in another country, where the owner of the original trade mark plans to expand to. This is done with the intent to sell the rights to the trade mark in the new country to the owner of the trade mark in the old country. However, she also recognises that legal instruments are usually in place to resolve such issues, which nonetheless do not resolve it without a noticeable loss of efficiency due to administrative and procedural costs in addition to the delay of litigation or negotiation. Despite this, the author highlights the importance of being able to 'reserve' a trade mark in order to secure the benefits from up front investments in a new brand, also arguing along the lines of Landes and Posner (1987) that the set of available names, signs, shapes etc. is far from being empty at the least, if not infinite.

In his reflections on the Protocol Relating to the Madrid Agreement Concerning the International Registration of Marks of 1989 (hereafter: Madrid Protocol), Schulte (1995a,b) notes that the level of scrutiny of trade mark application examination varies between two extremes across countries, i.e., from a mere formality check of the application documents to a thorough search for existing marks and the opportunity to oppose an application. Unfortunately without presenting any evidence, the author states that countries with the lowest level of scrutiny have the most crowded trade mark registers. The author also raises the question whether it is the office's responsibility to ensure integrity of the register or to protect prior trade mark rights. In an 
international context, each national trade mark office assumes a 'gate keeping' function, which, in a perfect world, prevents the international trade mark register from being cluttered with unused marks and guarantees that each trade mark entering the register meets the minimum standards common to all members of the international agreement. However, this is clearly not the case with France being one of the easiest jurisdictions in which to acquire a trade mark due to only minimal examination procedures and the US on the other side, having the most difficult trade mark system to acquire a mark. It follows that with entering an agreement such as the Madrid Protocol, countries with higher trade mark registration standards will experience an influx of trade marks from countries with lower standards, which again likely increases search, administration and litigation costs.

Another interesting point raised by the author is the fact that in the US the scope of the trade mark is limited to the good or service it is actually used for, contrary to most registration-based countries, where applicants can indicate any number of classes they wish to obtain protection in. In the US the stricter enforcement of the use requirement makes such broad applications strategies riskier. The use requirement therefore ensures maximum availability of trade marks and also informs future trade mark holders about used trade marks, thereby avoiding future conflicts. However, he also observes that when filing abroad, US firms do apply for trade mark protection with a much broader scope, i.e. for more classes of goods or services, than their mark is protected at home. The ability to do so is so important to US firms that even if they could acquire international trade mark protection based on their domestic trade mark, i.e., with very limited scope, they would choose not do so and apply at the respective national offices for broader protection, despite the additional expenses incurred. This remark hints at something that requires further research, i.e., the incentive of firms to register more broadly than required for their current or intended use of that trade mark. In short, the author does acknowledge the looming danger of registration-based trade mark registers accumulating 'dead wood', thereby making it harder to clear a desirable trade mark, which in turn motivates an empirical investigation into the structure of registration-based trade mark registers.

In an article that is mainly concerned with the adverse effects of the discretion the EU leaves with its member states in the Trade mark Directive 2008/95 of 22 October 2008, Depo (2010) highlights the importance of Article 12(1) of that directive with regard to the already enormous costs for a thorough pan-European registrability and availability search for a new trade mark in the community. Although expenses for registering a community trade mark are lower than registering it with every national trade mark office in the 27 member states of the community, one also has to account for the fact that the validity of a community trade mark depends on it not being registered and used as a community or national trade mark in any one of the 27 member states. The author argues that due to the large number of trade marks registered with the national trade mark offices, OHIM and WIPO, the required search efforts for a valid trade mark are staggering. Yet, even the most meticulous search may not ensure the risk free use of a new trade mark, since an owner of a trade mark can never be certain that his mark has not been acquired through use by a someone different elsewhere before her application. The problem of conducting an efficacious search is aggravated by the existence of trade marks which are not used commercially, since those merely add to the number of trade marks that cannot be used or that need to be investigated individually for commercial use. 
However, even if a desired registered trade mark appears not to be used commercially, the potential applicant has to go through several administrative, and arguably legal steps, until the senior trade mark is revoked and the mark can be registered with the new owner. Since the directive leaves it to the national judicial apparatus to decide which case has legal standing and which does not, different interpretations of when trade marks are in conflict can lead to severe limitations of the cleansing property of Article 12(1). Such is the case in Poland, claims the author, where the Supreme Administrative Court has taken a rather restrictive view of when trade marks are in conflict with each other. Without going into too much detail, the author concludes that due to the difficulties in achieving legal standing of revocation demands before national courts in Poland, the country's trade mark register may be flooded with unused trade marks, creating significant hurdles for future applicants due to increased search costs.

\subsection{Background: UK and OHIM Marks}

This section briefly describes the characteristics and differences between the two types of marks used in this analysis: UK and Community trade marks.

A UK firm has three ways of obtaining trade mark protection in the UK. The cheapest method is to apply for a UK trade mark (UKTM) from the IPO, which costs $£ 200 .{ }^{16} \mathrm{An}$ alternative route is to apply for a Community Trade Mark (CTM). The CTM was introduced in 1996 and is more expensive at $€ 900,{ }^{17}$ but a CTM covers all countries in the EU. ${ }^{18}$ In both cases the initial registration lasts for ten years, at which time a renewal fee is payable ( 200 for UKTMs and $€ 1350$ for CTMs if done electronically). A third route is the so-called Madrid-System, already referred to above, which is administered by WIPO. A trade mark registered in any country that is party of the Madrid Agreement or the Protocol can be used to obtain an international trade mark, which is valid in all designated signatory states of the Madrid Agreement and/or the Protocol. ${ }^{19}$ For a firm to apply for an international trade mark, the company needs to already have registered the trade mark (or applied for) in the country in which it is registered. This means that if a company that is registered in the UK applies for an international trade mark, it will already have registered a UK trade mark with the IPO. ${ }^{20}$

A trade mark application has to specify a class in which the trade mark is to be used. ${ }^{21}$ Trade mark infringement occurs only if products can be confounded, which requires both the mark and the product of the infringing party to be similar to the protected mark and product. Overall, the legal framework for UKTMs and CTMs is largely the same, since UK's Trade Mark Act 1994 was based on the European Trade Marks Directive, hence the choice between the two comes to balancing off the costs versus expected benefits. An important cost factor in obtaining a CTM apart from fees is the need to conduct a Europe wide search for existing trade marks with which

16 Fee indicated as of January 2009.

17 The application and registration fee is $€ 900$ if the application is filed electronically and $€ 1050$ if not.

18 It is not possible to limit the CTM to certain EU member states. The application for a CTM can be filed via the UK Intellectual Property Office or the Office for Harmonization in the Internal Market (OHIM) in Alicante, Spain. If the application is made via the UK Intellectual Property Office, an additional handling fee applies.

19 The 'Madrid Agreement Concerning the International Registration of Marks' was signed in 1891, and the Protocol Relating to the Madrid Agreement, which was adopted in 1989, entered into force on December 1, 1995.

20 See the official website of the Madrid System: http://www.wipo.int/madrid/en/general/

21 Multiple classes per single application are allowed, although each additional category requires an additional fee of $£ 50$ in the case of UKTMs (http://www.ipo.gov.uk/types/tm/t-formsfees.htm). For CTMs, the basic fee covers three classes of goods and services. Each additional class costs $€ 150$ for application and registration. 
the firm's own mark could be confounded. As already mentioned above, the search costs increase with the size of the register. If a UK firm never expects to sell in the EU, then a UK trade mark is cheaper and will offer all the protection needed. If there is any expectation of sales in the EU then, as long as the firm can afford the greater costs, it should use Community trade marks. Also contrary to patents, a trade mark can be renewed indefinitely. Applications for trade marks are examined and then published, allowing a period of time for others to object, before full registration. In the case of UKTMs and CTMS, third parties have two to three months to oppose the registration of a trade mark after its publication. ${ }^{22}$ In the case of CTMs, the Community mark is only registered if there is no opposition from any of the member states. Hence, any valid opposition from a single member country prejudices the registration for the entire EU. ${ }^{23} \mathrm{~A}$ trade mark can also be invalidated after it has been registered if anyone successfully argues that it should never have been registered. Also, if the trade mark is not used for five years following its registration, anyone can apply to have the trade mark revoked. The average duration from the filing of an application to registration for UKTMs is six months if there are no objections or opposition. ${ }^{24}$ The delay in getting a CTM appears somewhat longer than for a UKTM at around 15 months. ${ }^{25}$

22 Usually, in the case of UKTMs, the UK trade mark office notifies the owners of existing trade marks if there is reason to believe that the new trade mark could be confounded with existing marks. The notified parties then have the right to oppose registration of the new trade mark. Other third parties are allowed to comment on published trade marks. However, if the comment relates to existing trade marks that the examiner has overlooked, this will also lead to notification of the existing owner of a trade mark who then has the opportunity to oppose the registration.

23 We analyse oppositions in Section 3.2.3.

24 http://www.ipo.gov.uk/t-essentialreading.pdf

25 http://oami.europa.eu/en/mark/default.htm 


\section{Part II - Empirical Analysis}

The following sections present a number of different attempts to learn about cluttering from existing trade mark data bases. The novelty of the question we analyse makes it important to approach the problem from different angles. For instance cluttering might be a general phenomenon or it might be restricted to specific parts of the trade mark system, either specific firms or specific markets. Cluttering might be the consequence of unused trade marks or of overly broad trade marks.

Each section below contains a discussion of how the particular analysis presented relates to the problem of cluttering and what it shows.

\subsection{Analysis of UK Data}

\subsubsection{UK Data - OFLIP}

To provide a first empirical investigation into the possibility that trade mark registers are cluttered, we created an unbalanced firm-level panel for the period 2000-2007 using trade mark data from the Oxford Firm Level Intellectual Property (OFLIP) database and financial data from Financial Analysis Made Easy (FAME), provided by Bureau van Dijk. The panel contains 201,021 observations, covering 27,161 firms registered in the UK with at least three consecutive observations for each firm.

The OFLIP database provides information on UK trade mark publications and Community trade marks registered for each firm in FAME (see Helmers et al. (2011), for further details). One issue of relevance is that while firm-level trade mark data is straightforward and intuitive in the case of stand-alone firms, the situation is more complex when firms are part of business groups. Throughout this study we use unconsolidated IP data, that is, we do not reassign trade marks across firms within the same business group. ${ }^{26}$

\subsubsection{Descriptive Analysis}

In what follows, a descriptive analysis of the distribution of trade marks and the use of Nice classes by firms registered in the UK for the period 2000-2007 is provided. Studying the relation between the number of trade marks applied for by UK firms and the number of Nice classes ${ }^{27}$ covered by each trade mark yields insights into whether UK applicants to the IPO behave differently from UK applicants to OHIM, and whether their behaviour has changed over time.

26 One could, for example, allocate all trade-marks held by subsidiaries to the holding company and vice versa. We do not undertake such consolidation to avoid any arbitrary decision that this reassignment would imply in the absence of ancillary information on firms' strategies and procedures in dealing with IP.

27 The Nice Classification of Goods and Services is a list of 45 classes of goods and services, thousands of specific types of each class and detailed description of most of those. It is currently in use by 148 countries. It was agreed in 1957 and is updated every 5 years. 
If there were significant cluttering of the trade mark register we might be able to observe an increase in average trade mark applications per firm or in the average number of classes each trade mark is registered in over time. Taking advantage of the information in the OFLIP data base, the development over time of the average number of trade marks per firm and of the average number of classes per trade mark will be illustrated for each of 13 aggregated industries and for each firm size category.

We find that the number of Nice classes per trade mark is increasing over time. This increase is most pronounced for medium and large enterprises. We do not observe UK firms applying for more trade marks over time, regardless of the sector or the size class of the firms.

\section{Trade Marks per Firm}

- By Industry: Figure 2 shows the average number of trade mark applications over the period 2000-2007 across 13 aggregate industries ${ }^{28}$ applied for by UK firms at the IPO or OHIM. Leaving aside the Communications industry, there is very little variation over time or across industries. On average firms apply for more trade marks at IPO that at OHIM. Furthermore, the average number of IPO trade mark applications varies between two and six per year, while the number of applications at OHIM varies between one and four per year. Firms in the Communications industry file significantly more trade mark applications in every year, with a peak in 2001 (14 TMs at IPO, 7 at OHIM) and again in 2007 (13 and 6, respectively), and between four and six applications per year between those peak years. Thus, apart from the activities in the Communications industry, there is no evidence for cluttering from this perspective. This means that there are no trends suggesting that all firms are applying for more trade marks over time at IPO or OHIM. These averages might still be hiding specific groups of firms that are behaving differently.

- By Firm Size Category: Turning to an analysis of the number of trade marks per firm within the different firm size categories (micro, small, medium and large) Figure 3 shows that as firms get larger they file for more trade marks. The graphs also show that recently there have been slight increases in the average number of trade marks per firm by small, medium and large firms. However, these increases are not very significant at present.

Distribution of Trade marks over Nice classes - This measure looks at the relative distribution of trade marks over the space of the 45 Nice classes to uncover Nice classes with an exceptionally large number of registered marks. Since the distribution of OHIM trade marks over Nice classes does not differ noticeably from the distribution of IPO trade marks over classes, the discussion concerns the joint distribution. Due to their size, the figures discussed below can be found in the Appendix.

28 The Electric, Gas and Water industries have been dropped from the analyses as they contained extreme outliers, with firms as for instance NPower filing up to 630 trade marks in one single year due to numerous merger, restructuring and renaming activities during the period of investigation 


\section{Figure 2: Distribution of firms' average number of trade marks by sector.}

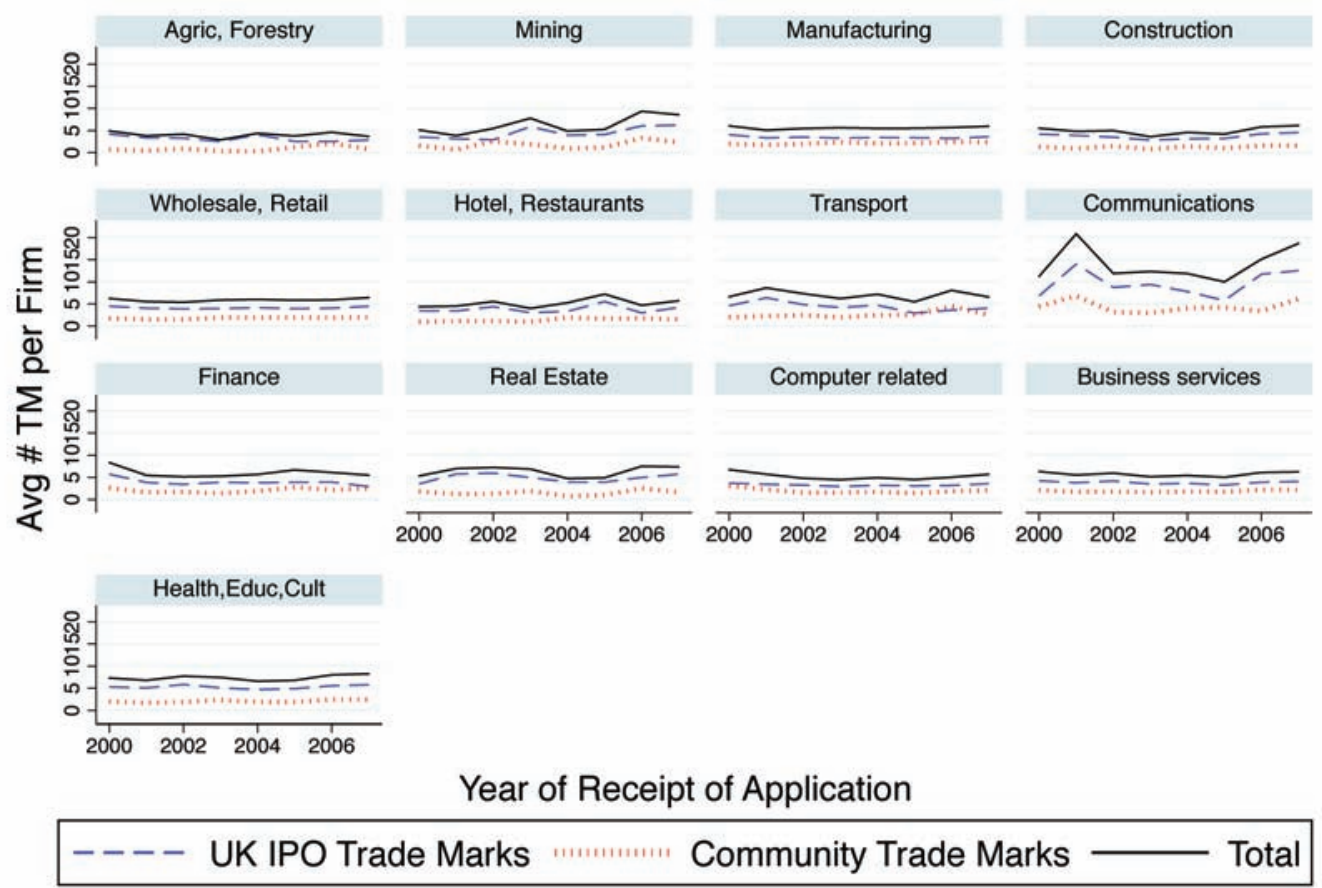

All graphs are based on OFLIP and FAME data. Graphs are by sector.

Figure 3: Distribution of firms' average number of trade marks by firm size.

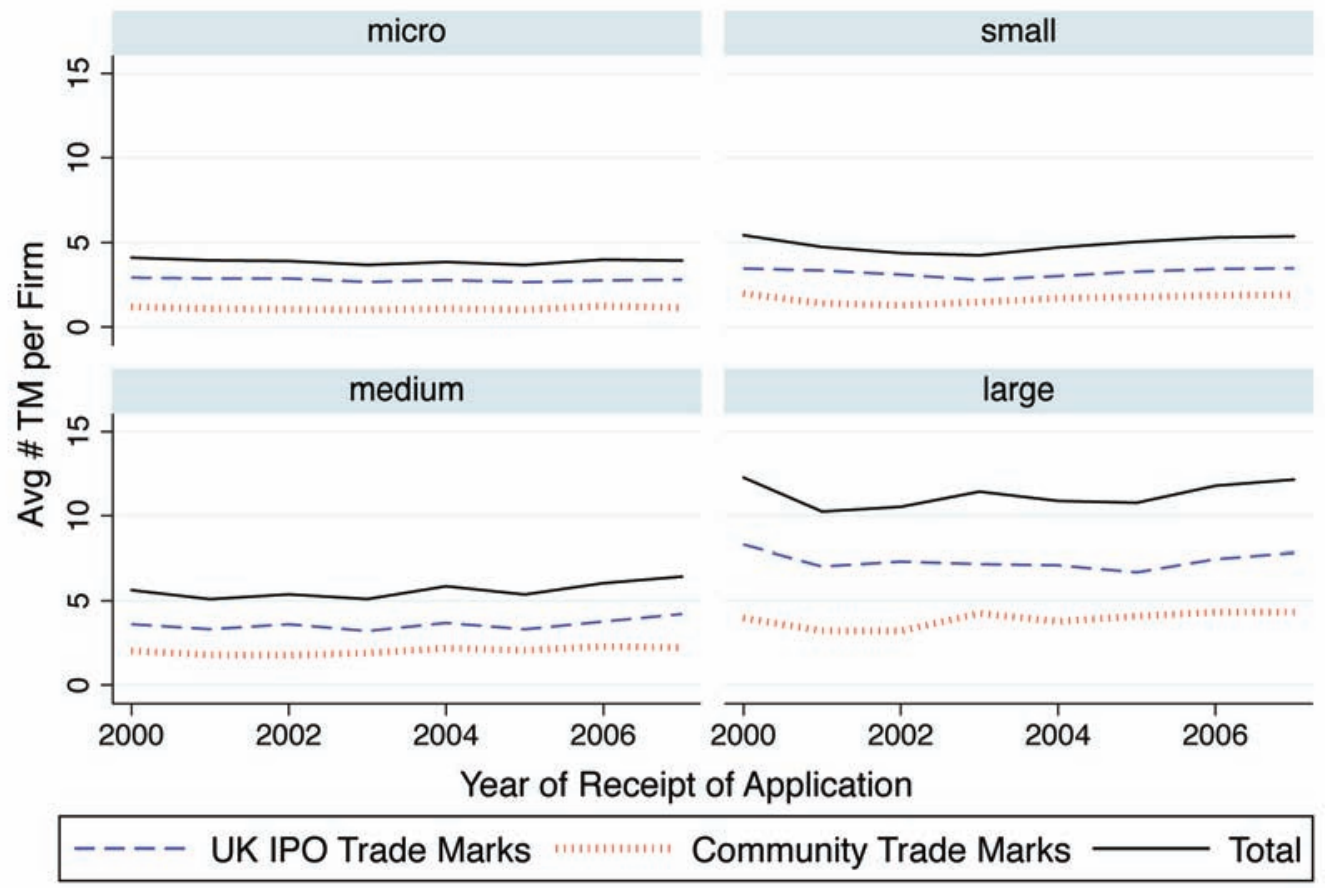

All graphs are based on OFLIP and FAME data. Graphs are by firm size. 
- Sector: Figure 13 shows the aggregated distribution of trade marks over Nice classes across five aggregate sectors. It is noteworthy that over a quarter of all trade marks from the high tech sector are filed for class 9, scientific apparatus and another $15 \%$ in class 42 (scientific and technological services). Furthermore, firms in the R\&D sector file large shares of their trade marks in class 5 (pharmaceuticals) and again 9 and 42 . One can also see that medium-tech firms tend to file relatively more trade marks than firms in other sectors in classes 1-3 (chemicals, paint and laundry), 5, 7 (machines and machine tools) and 11 (lighting and heating). Firms which fall under the category 'Other Manufacturing' file more marks than others in classes 6 (common metals), 19 (building materials), 20 (furniture), 25 (clothing, footwear), 29 and 30 (meat, fish, and coffee and salt).

- Firm size category: Figure 14 shows the aggregated distribution of trade marks over Nice classes across the different firm size categories. Almost half of all trade marks are filed for classes $9,42,35,16,41$ and $25 .{ }^{29}$ As regards to the distribution across firm size categories, large firms have the relative lead in classes $29-33,36-40,43-45,{ }^{30}$ while micro firms file more of their trade marks than others in classes $3,9,16,25,28,35,41$ and 42 . The remaining classes are predominantly aimed at by small and medium firms, which show a very similar filing pattern with the exception of a few classes $(9,19,20$, $35,41,42)$.

- Over time: Figure 15 shows the aggregated distribution of trade marks over Nice classes in each year of the period under investigation. It is interesting to note that the share of most of the large classes $(9,16,41,42)$ decreases over time. Trade mark filings for Advertising and Business Management (class 35 ) show a U-shape over the eight-year period, while trade marking for clothing (25) increased slightly. The relatively large drop in the share of TM filings in the mentioned classes leads to increasing shares of most of the remaining classes. It cannot be observed that firms moved to any particular class.

This analysis reconfirms what we have found above. There is no clear evidence that cluttering in the UK consists of more trade marks per firm. What we find here is that if we disaggregate trade mark applications by Nice classes there is evidence that some Nice classes are becoming proportionately less important whilst others are growing in importance (Figure 15). We can observe that this is partly driven by a shift towards the new service classes (43-45). It may be interesting to note that the pharmaceuticals classes are generally unaffected by this trend. Their share of overall trade mark applications has increased. We will come back to this point further below.

29 9=Scientific apparatus, 42=other services, 35=Advertising and Business Management, 16=Paper and Packaging, $41=$ Sports \& Education, $25=$ Clothing

30 See appendix for full list of class descriptions 
Average Number of Nice classes per Trade Mark - This measure indicates how many Nice classes, on average, are registered for each trade mark. We cross-tabulate this information in two dimensions:

- By Industry: While the average number of trade marks per firm does not vary a lot across sectors or over time, in terms of the average number of classes each trade mark covers, Figure 4 shows a lot of variation across sectors, between IPO trade marks and community trade marks and also over time. Overall, one can see that across all sectors, firms seek protection in more Nice classes for each OHIM trade mark (approximately 3 classes on average) compared to IPO trade marks (2). In most sectors this difference is subtle, with the exception of the Mining, Construction, Hotel and Restaurants as well as the Real Estate sectors. In the Mining sector between 2002 and 2005, firms applying for a community trade mark registered their mark for two more classes (4 on average) than firms that applied for IPO trade marks. However, this number drops back to the level of classes for IPO trade marks (2) on follows it from there on. A similarly non-persistent peak in the number of Nice classes per community TM application can be found in Construction in 2002, in the Hotel and Restaurant industry in 2004 and in Transport in 2005. Firms in the Construction, Communications as well as Health, Education and Cultural industries increase the number of classes in use over time.

- By Firm Size Category: Figure 5 shows the average number of Nice classes per trade mark across the different firm size categories. Firms of all sizes file their trade marks for more classes at OHIM than at IPO. Furthermore, the number of classes per trade mark is slowly increasing over time, thus providing some indication of cluttering. Again, the average number of Nice classes per trade mark for micro, small and medium firms varies between 2.1 and 2.5 classes for an IPO trade mark and 3 classes for a community trade mark. However, large firms, on average, file between 2.5 and 3 classes per IPO trade mark compared to 3 to 3.5 classes per trade mark at OHIM, which likely corresponds to the larger and increasing number of products/services on offer.

This section shows that there is some indication that medium and large firms are tending to apply for more Nice classes per trade mark than in the past. This might be an indication of cluttering, it may also be entirely innocuous. For instance it might be due to increased product diversification. Future research will have to show what is driving this development. 


\section{Figure 4: Distribution of average number of Nice classes per trade mark by sector.}
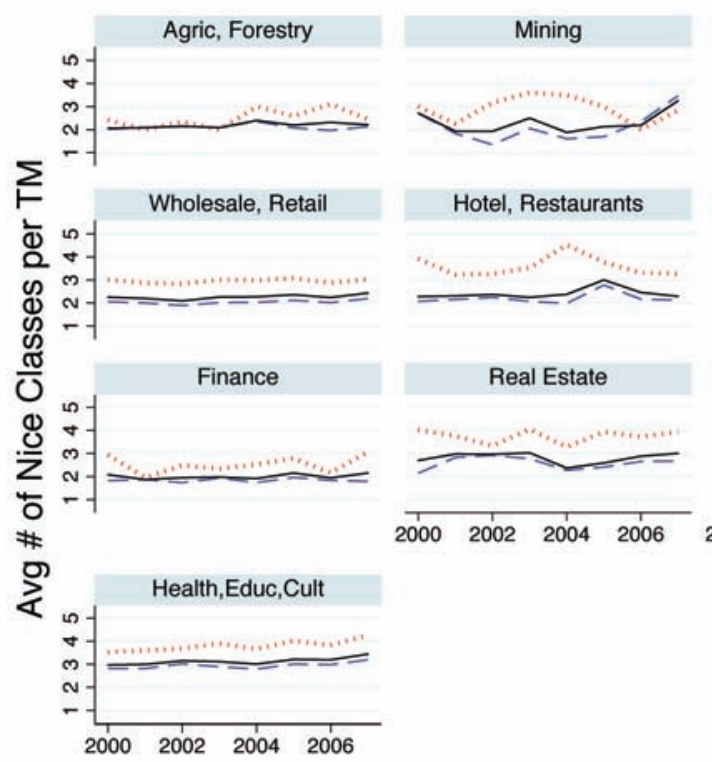

Manufacturing

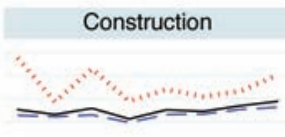

Hotel, Restaurants

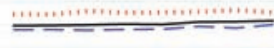

Communications

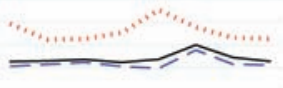

Transport

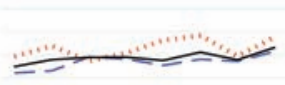

Real Estate
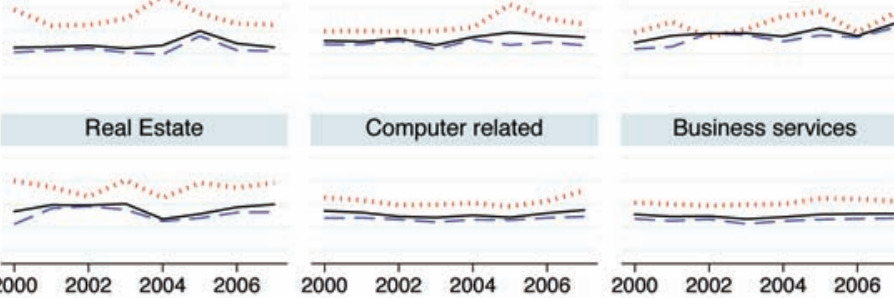

Business services

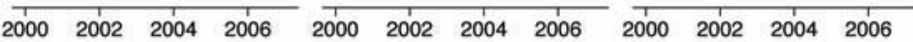

$20002002 \quad 2004 \quad 2006$

Year of Receipt of Application

\section{- - - UK IPO Trade Marks $\ldots \ldots \ldots \ldots \ldots$ Community Trade Marks}

Average

Graphs are based on OFLIP and FAME data. Graphs are by sector.

\section{Figure 5: Distribution of average number of Nice classes per trade mark by firm size.}

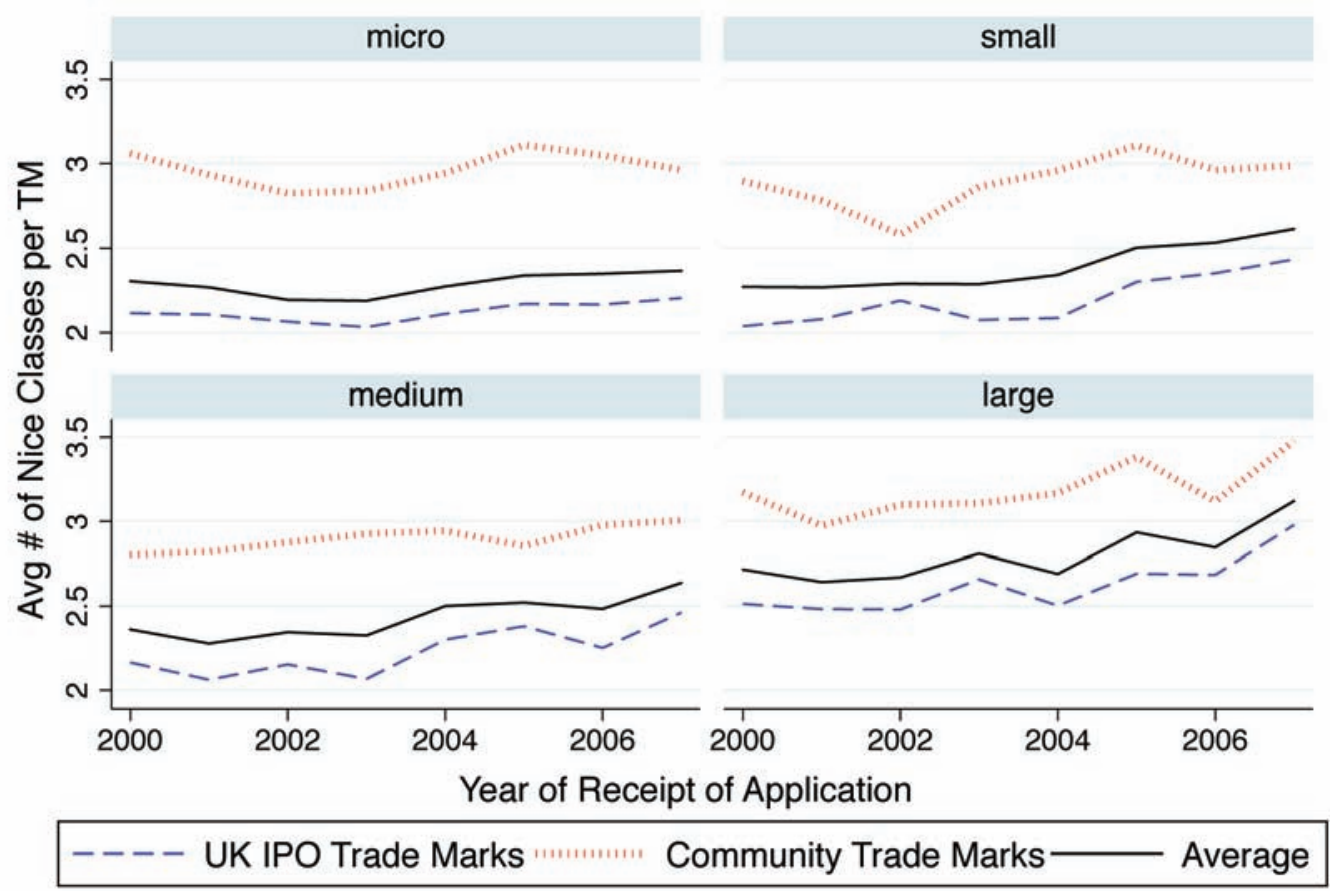

All graphs are based on OFLIP and FAME data. Graphs are by firm size. 


\subsubsection{Growth Regression}

The preceding graphs provide information on the distribution of trade marks across Nice classes and on how many Nice classes the average trade mark covers. A number of patterns show through these figures. Most are to be expected: for example, firms in the high-tech sector file every fourth trade mark in the class for scientific apparatus. However, some are less obvious. For example, the fact that firms across all size categories and sectors appear to move away from filing in what may be 'focal classes' (i.e., class 9, 16, 25, 35, 41 and 42) towards a broader filing pattern.

To gain additional insight into the structure underlying these figures, we employ a simple regression model. We define indicators of potential cluttering in order to gauge the existence and extent of trade mark 'cluttering'. OFLIP allows us to interrelate changes in a firm's total assets over time (across firm size categories and industries) and the change of a firm's trade mark filings and stock. We test for trade mark cluttering by looking for an association between growth in firm size and growth in trade mark filings. If the number of filings or the stock of trade marks grew disproportionately faster than assets, this may be interpreted as evidence for cluttering. Hence, we test whether growth in assets predicts growth in trade mark filings. In order to analyse this in the data, we estimate the following model (in levels and first differences):

$$
\Delta T M_{i j, t}^{a p p s}=\triangle A_{i j, t}+T M_{i j, t-1}^{s t o c k}+r_{i j, t-1}^{o p p}+r_{j, t}^{o p p}+T M_{j, t}^{a v g}+D_{t}+D_{j}+\varepsilon_{i j, t}
$$

In this regression, we index an individual firm with $i$ and the different industries by $j$. Thus, $\triangle T M_{i j, t}^{a p p s}$ denotes the change of the number of trade mark applications by firm $i$, which is in industry $j$, from last period $t-1$ to this period $t . \triangle A_{i j, t}$ is the change in firm $i$ 's assets from last period $(t-1)$ to this period $(t), T M_{i j, t-1}^{\text {stock }}$ is firm $i$ 's stock of trade marks in $t-1$, $r_{i j, t-1}^{o p p}$ is firm $i$ 's lagged opposition rate, i.e. the number of oppositions a firm received last period divided by the number of trade marks it had filed. In the same vein, $r_{j, t}^{o p p}$ is the average opposition rate in the firm's industry $j$, and $T M_{j, t}^{a v g}$ represents the average number of trade marks filed in firm $i$ 's industry $j$. $D_{t}$ and $D_{j}$ are time and industry dummies, respectively. The year dummies control for time-specific effects common to all firms, i.e. changes in the economic environment in a given year which affects all firms in the same manner. Similarly, the industry dummies control for time invariant, industry specific effects. Differently put, if there are differences between industries, which do not change over time, they will be captured by these variables. Table 1 below presents the results. The table contains results from four different specifications.

Specification (1) is the baseline, only including total assets in levels as regressor, which in this case is positive and significant, implying that larger firms (measured by assets) are associated with a faster growing trade mark portfolio.

Specification (2) regresses changes of the number of trade mark applications from one period to the next on changes in a number of explanatory variables. In this specification, everything that is constant from one period to the next drops out.

The coefficient on the change in assets is very close to zero but statistically significant, yet negligible in magnitude: a change in assets of one billion GBP would lead, on average, to 0.004 additional trade mark applications. 


\section{Table 1: Trade Mark Portfolio Growth Regressions}

\begin{tabular}{|c|c|c|c|c|}
\hline & $\begin{array}{l}(1) \\
\text { Base }\end{array}$ & $\begin{array}{l}\text { (2) } \\
\text { All FD }\end{array}$ & $\begin{array}{l}\text { (3) } \\
\text { FD } \\
\text { Assets }\end{array}$ & $\begin{array}{l}\text { (4) } \\
\text { FD Assets \& } \\
\text { Controls }\end{array}$ \\
\hline Total Assets in Bn GBP & $\begin{array}{l}.0066^{* *} \\
(.0023)\end{array}$ & & & \\
\hline Avg \# of TM Apps in Industry & & & & $\begin{array}{l}.5^{*} \\
(.22)\end{array}$ \\
\hline Lagged TM Stock & & & & $\begin{array}{l}-.0027 \\
(.011)\end{array}$ \\
\hline Lagged Rate of Received Opp/TM filings & & & & $\begin{array}{l}-2.9^{* * *} \\
(.17)\end{array}$ \\
\hline Industry Opposition Rate & & & & $\begin{array}{l}.97 \\
(.82)\end{array}$ \\
\hline First Difference Assets in Bn GBP & & $\begin{array}{l}.0044^{* *} \\
(.0016)\end{array}$ & $\begin{array}{l}.0021 \\
(.0073)\end{array}$ & $\begin{array}{l}.0028 \\
(.0073)\end{array}$ \\
\hline FD Avg \# of TM Apps in Industry & & $\begin{array}{l}.98^{* * *} \\
(.074)\end{array}$ & & \\
\hline FD Lagged TMstock & & $\begin{array}{l}.00029 \\
(.00084)\end{array}$ & & \\
\hline FD Lagged Rate of Received Opp/Tm filings & & $\begin{array}{l}-1.3^{* * *} \\
(.042)\end{array}$ & & \\
\hline FD Industry Opposition Rate & & $\begin{array}{l}.13 \\
(.14)\end{array}$ & & \\
\hline 2002 & & & $\begin{array}{l}.12^{* * *} \\
(.026)\end{array}$ & $\begin{array}{l}.13^{* * *} \\
(.028)\end{array}$ \\
\hline 2003 & & & $\begin{array}{l}.12^{* * *} \\
(.023)\end{array}$ & $\begin{array}{l}.11^{* * *} \\
(.026)\end{array}$ \\
\hline 2004 & & & $\begin{array}{l}.14^{* * *} \\
(.022)\end{array}$ & $\begin{array}{l}.16^{* \star *} \\
(.024)\end{array}$ \\
\hline 2005 & & & $\begin{array}{l}.13^{* * *} \\
(.023)\end{array}$ & $\begin{array}{l}.13^{* * *} \\
(.027)\end{array}$ \\
\hline 2006 & & & $\begin{array}{l}.2^{* * *} \\
(.023)\end{array}$ & $\begin{array}{l}.16^{* * *} \\
(.041)\end{array}$ \\
\hline 2007 & & & $\begin{array}{l}.14^{* * *} \\
(.024)\end{array}$ & $\begin{array}{l}.16^{* * *} \\
(.034)\end{array}$ \\
\hline Constant & $\begin{array}{l}.61^{* * *} \\
(.011)\end{array}$ & $\begin{array}{l}-.01 \\
(.0061)\end{array}$ & $\begin{array}{l}-.13^{* * *} \\
(.029)\end{array}$ & $\begin{array}{l}-.36^{\star *} \\
(.13)\end{array}$ \\
\hline$N$ & 201021 & 145448 & 161195 & 161195 \\
\hline
\end{tabular}

Standard errors in parentheses: * $p<0.05,{ }^{* *} p<0.01,{ }^{* * *} p<0.001$

FD $=$ First Differences

Dependent variable: change in trade mark applications or in the case of FD the rate of change of TM applications. 
The impact of a change in the number of average industry-level trade mark applications is far more efficacious. It is almost unity and statistically significant at the $1 \%$ significance level. In other words, a firm's trade marking behaviour correlates strongly with intra-industry filing behaviour, i.e. if other firms in industry j file one additional trade mark on top of the number they usually apply for on average per year, firm i likely also files an additional trade mark on top of the number of trade marks it would have applied for otherwise. The change of period's $t-1$ trade mark stock has no impact on the number of applications filed in period $t$.

Furthermore, a change of the rate of received oppositions in the last period has a significantly negative impact exceeding one (-1.3) on the number of trade mark applications in this period. This shows that if a firm encounters a growing number of legal conflicts concerning last period's trade mark applications, it reduces its level of applications this period.

Estimating the impact of a change of assets on the change of the number of trade mark applications including time and industry dummies (Column (3) in Table 1) shows that there is a weakly upward sloping time trend with a peak in 2006. The change of assets in this and specification (4) has no significant impact on the change of the trade marking activity. However, including the industry average of TM applications in levels (specification (4)) again has a significant effect in the same direction as in specification (2), i.e., an increasing industry trade marking activity leads to more trade mark applications. Since the industry wide average is now measured in levels, the coefficient of 0.5 implies that on average, firms in industry $j$ need to apply for two more trade marks in order for firm i to also file an additional application. Including last period's trade mark stock in levels remains insignificant, while the lagged rate of oppositions received is again negative and significant at the $1 \%$ level. Intuitively, the coefficient of -2.9 for the lagged rate of own opposition says that on average, if all of firm i's trade mark applications in the last period had been opposed, firm $i$ would file 2.9 fewer applications this period (if possible).

Robustness - The same regressions were undertaken using the change of the number of classes as dependent variable, but qualitatively results remained the same. Furthermore, in addition to industry dummies, covariates representing the distribution of a firm's trade mark activity over Nice classes were included, all of which turned out to be statistically significant. However, the findings on the explanatory variables used in the original model and presented in the table above did not change, confirming the robustness of the chosen specifications.

Due to the inclusion of the lagged trade mark stock these regressions can be considered as variants of dynamic models. These introduce complex statistical problems which we do not focus on here. As a robustness check we have also estimated the models reported here as fixed effects models. The results we obtained from these were very similar to those reported above. This suggests that the dynamics do not introduce biases that affect our results.

\subsubsection{Summary}

This section presented descriptive evidence of the trade mark activity by UK firms as captured by OFLIP and additional data on oppositions from OHIM and the IPO. For our sample covering the period 2000-2007, large firms, on average, file in more classes (3) compared to micro, small and medium sized firms (2.5). More striking, however, is the fact that across all firm sizes and industries, firms file for one more class, on average, at OHIM than they do at the IPO. Looking across industries, we find remarkably little variation in the number of classes firms file in, with the exception of Mining, Construction as well as Hotels and Restaurants. 
The analysis of the distribution of trade mark activity over classes shows that there exist a number of 'focal classes', i.e., classes 9, 16, 25, 35, 41 and 42. Over time, firms appear to file more widely across Nice classes.

Most importantly, we find that the number of Nice classes firms apply for per trade mark increases for medium sized and large firms. This suggests that such firms could be contributing to cluttering of trade mark registers. However, the present analysis is not sufficiently detailed to exclude alternative explanations.

Using a simple linear regression model, we show that trade mark growth is largely independent of a firm's asset growth, while the average industry trade marking activity and the number of oppositions a firm receives predict in a statistically significant way the number of trade mark applications in a given year.

The effect of average industry trade marking activity is particularly relevant in the context of this study. It shows that firms' respond to increases in their competitors' trade marking activities by increasing their own level of trade mark applications. In other words there is evidence of a feedback mechanism. This feedback mechanism is a precondition for cluttering to become systemic. It indicates that excess trade mark applications by some firms can provide incentives for more applications by their direct "neighbours" in the trade mark system.

These regressions do not show that there are unused trade marks, nor whether their numbers are increasing or decreasing. Such regressions can only be provided if we improve our measures of use of trade marks in future work.

\subsection{Analysis of OHIM Data}

In this section we use register data obtained from OHIM to further analyse whether there is evidence of cluttering of trade mark registers in the United Kingdom as well as in Europe. The data we use for analysis of OHIM trade marks contains much more information on procedural matters than OFLIP which we have used for the analysis of UK trade marks in Section 3.1 above. However, this data set contains no information on firms. Thus this section can broaden our understanding of cluttering in important ways but it also has limitations ${ }^{31}$

Trade marks registered by OHIM are effective throughout the EU, thus this office represents an important alternative to IPO for any firm doing business in the United Kingdom. This is a further reason to analyse $\mathrm{OHIM}$ data in the context of this report.

This section consists of four subsections. In the following subsection we provide a brief overview of findings from a previous report written for the European Commission on OHIM and trade mark registration there. Most importantly this section contains evidence from a survey of users of the European trade marks system on the effects of the current size of trade mark registers. The evidence shows that a majority of agents representing trade mark applicants believe that trade mark cluttering is a problem.

31 For instance we are unable to determine how firm size or sector specific effects affect trends at the OHIM level. 
Then in Subsection 3.2.2 we briefly analyse the effects of OHIM's pricing structure on the breadth of trade mark applications. In contrast to many national trade mark offices OHIM charges the same application fee for applications registered in 1 to 3 Nice classes. If the applicant wishes to protect their mark in further classes, costs increase linearly after the third class. This pricing scheme provides strong incentives to protect exactly three classes.

This subsection shows that applicants respond to the pricing scheme and frequently apply in three Nice classes. Using data on opposition cases we are also able to show that firms are more likely to have their marks restricted or partly rejected when they apply for exactly three Nice classes, than when they apply for more or fewer classes. This indicates that the 3 for 1 policy at OHIM contributes to cluttering.

Next Subsection 3.2.3 exploits the abolition of refusals on the basis of relative grounds examination (RGE) in the United Kingdom in October of 2007 to test whether there is evidence that RGE by IPO affected the ability of firms to register trade marks that are more similar to existing registered marks. We do this by comparing how opposition to trade marks registered by UK firms changed at OHIM and IPO before and after October of 2007.

This subsection provides results that suggest UK applicants were avoiding RGE by applying for trade marks at OHIM. By doing this the applicants were more likely to have their trade marks opposed. If the results from this subsection can be further corroborated in future work we will learn how valuable RGE is in preventing clutter.

Then, in subsection 3.2.4 we provide results from ongoing research that suggests that Nice classes used to protect pharmaceutical trade marks are affected by cluttering through excessive applications. The evidence in this case is drawn from the policy shock that was European enlargement in 2004.

This subsection demonstrates how different regulatory regimes that simultaneously affect trade mark applicants can provide incentives for cluttering. The section also demonstrates that simultaneous applications are one mechanism through which applicants can contribute to cluttering. At present the evidence is limited to pharmaceutical firms as we do not have sufficiently good data to determine whether there is cluttering through simultaneous applications by other firms. 


\subsubsection{Trade marks at OHIM}

This Subsection consists of two parts. The first contains general statistical information from register data. The second contains survey results from a survey of users of the European trade mark system.

Statistical Background Information - First, we provide a brief summary of applications, registrations and oppositions at OHIM. We compare applications from the United Kingdom to all applications. The section provides some background information for the analysis of opposition rates that follows below.

Figure 6 below provides an overview of the demand for trade marks at OHIM. It also contains information about the level of registrations and oppositions.

Figure 6: Demand for the Community Trade Mark

Trade Mark Applications, Registrations and Oppositions

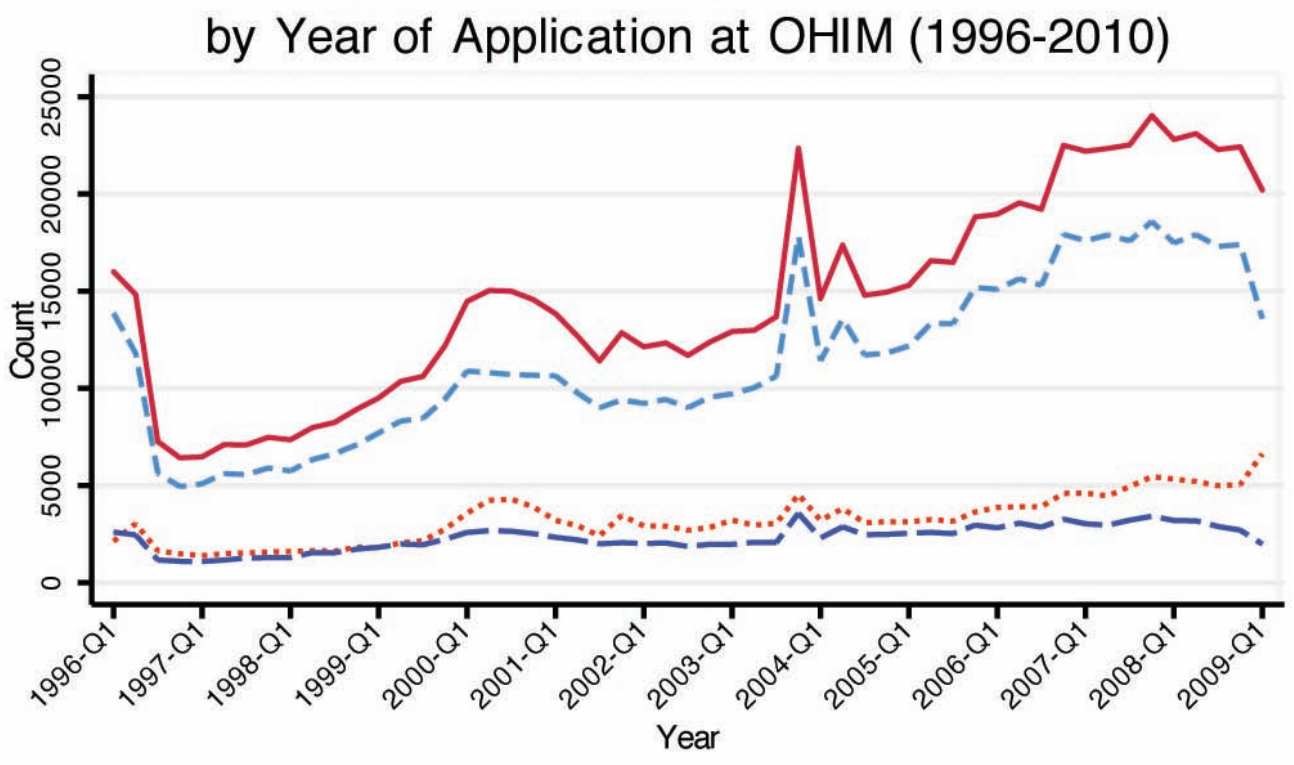

— Applications - - - Registrations ......... Not Registered _ — . Oppositions

Note: Based on data supplied by OHIM in January 2010 and own calculations

There are two main facts to note here:

- Demand for the community trade mark has been strong and increasing since OHIM began registering trade marks in 1996. Slightly more than 5000 applications made in the first quarter of 1997 were eventually registered at OHIM. Ten years later substantially more than 15,000 applications per quarter were eventually registered. 
- The level of oppositions has mimicked applications since 1996. The overall opposition rate at OHIM is quite stable around $16.6 \%$ in the years preceding 2007. Table 3 further below provides annual information in column 2 .

Figure 7 below provides an overview of demand for trade marks at OHIM that originate in the UK. The figure also provides information about registrations and oppositions. Comparing demand for OHIM trade marks from UK companies to overall demand we note three facts:

- Demand for OHIM trade marks from the UK did not grow quite as fast as the overall demand for OHIM trade marks.

- Opposition to UK applicants' marks at OHIM is stable at around $18.2 \%$ before 2007. Table 3 further below provides annual information in column 3 .

- UK demand for OHIM trade marks was more pronounced during the dot.com boom years than average demand and less so before the 2004 expansion of the EU. This presumably reflects the composition of UK firms and also the extent to which these firms trade with the countries that joined in 2004.

\section{Figure 7: UK Demand for the Community Trade Mark}

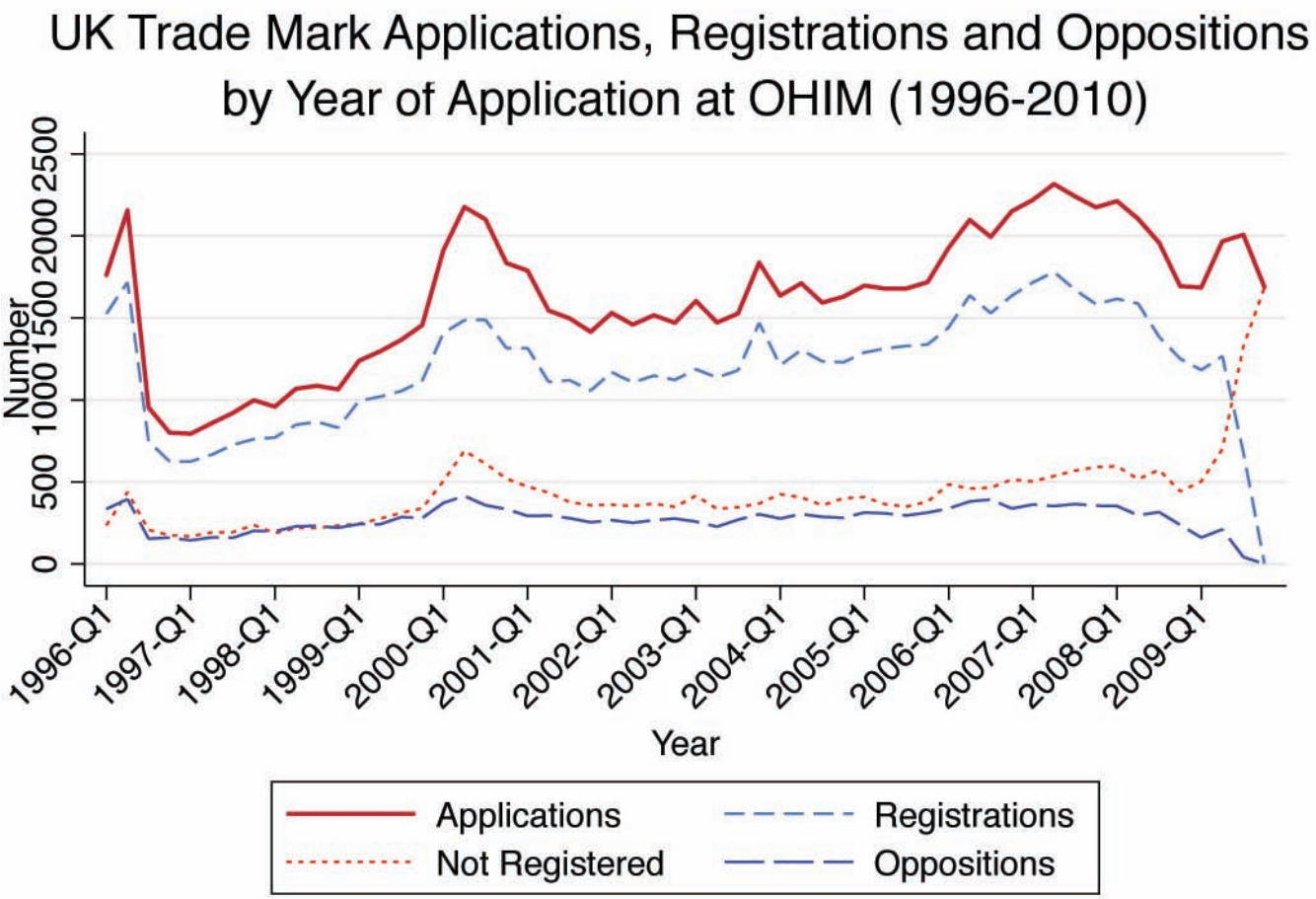

Note: Based on data supplied by OHIM in January 2010 and own calculations 
Results from the Allensbach Survey of Users - The only direct evidence on cluttering available so far comes from a survey conducted by the Institut fur" Demoskopie Allensbach and the Max Planck Institute for Intellectual Property, Competition and Tax Law (2010) (Kur et al., 2011). The survey was conducted in 2010 and investigates the use of the Community trade mark system. The survey is based on a random sample of 1,599 users where responses are separated for trade mark owners and trade mark agents/attorneys. One of the questions put to users was about the effects of the large number of trade marks on the register administered by OHIM.

Q16: Which of the following two opinions do you share?

a) The current number of CTMs that are either not used at all or only for some of the goods and services listed is tolerable and is therefore not a problem.

b) In the meantime, there are too many CTMs in the register that are either not used at all or only from some of the goods or services listed, and this is a problem.

c) No opinion/impossible to say.

Among trade mark users/owners, only about $21 \%$ consider cluttering to be a problem whereas $29 \%$ do not consider this to be an issue. In the UK, the figures are $17 \%$ and $27 \%$. It turns out that there are no noteworthy differences across firm size categories or sectors of activity. Yet, the picture changes substantially when we look at the responses given by trade mark attorneys/ agents. Here, overall $41 \%$ consider cluttering to be a problem and only $33 \%$ do not. This difference is even more pronounced when looking at the UK: $54 \%$ of trade mark attorneys consider cluttering to be a problem and only $14 \%$ do not. This difference of 40 percentage points is by far the largest among the European countries covered by the survey. The study also provides a number of excerpts of more detailed responses. An anonymous UK trade mark agent summarizes the view expressed by the high share of agents considering cluttering to be a problem as follows: it is extremely difficult to do a sensible clearance search as there are so many CTMs that are not used or ones which are registered for class headings so that there is no indication of what the likely commercial interest is.

\subsubsection{The Breadth of Trade Mark Applications}

As noted in the introduction to this section OHIM's pricing schedules create strong incentives for firms to protect their marks in three Nice classes as long as the applicant does not seek protection in more classes. This is because protection in three Nice classes is no more expensive than protection in one or two Nice classes. If trade mark applications are regularly excessively broad this will raise the costs of search for later applicants.

Protecting a trade mark in more than one class is advantageous if the applicant expects to use the trade mark in several different product markets. As trade marks cannot subsequently be broadened, it may be that some applicants choose to make their trade mark applications broader than is necessary in the short run. This would provide them with the option of extending business into markets (classes) in which their marks are already nominally protected. ${ }^{32}$

32 As long as a mark is not a well known mark, non-use of the mark in a specific market (class) will usually mean that the applicant cannot enforce the mark in that market (class). 
Broadening protection of marks beyond classes in which use can be demonstrated is risky because non-use in specific classes can lead to legal challenges. Evidence from the opposition

Analysis provided further below shows that adding additional classes to an application increases the probability of opposition to that application significantly. This suggests that court cases are also more likely to affect marks that are overly broad. One might expect that this serves to check firms' incentives to apply for protection more broadly than is strictly necessary. We briefly provide some evidence on this matter below.

In this section we analyse how the costs and benefits of adding additional Nice classes to an application affect firms' application behaviour. This section is descriptive. We cannot rule out that what we depict is the result of firms' market behaviour rather than the result of the incentives created by the pricing scheme implemented by OHIM. However, the evidence does not suggest this is the case.

\section{Figure 8: Histograms of number of Nice classes on IPO and OHIM trade mark}

\section{applications}
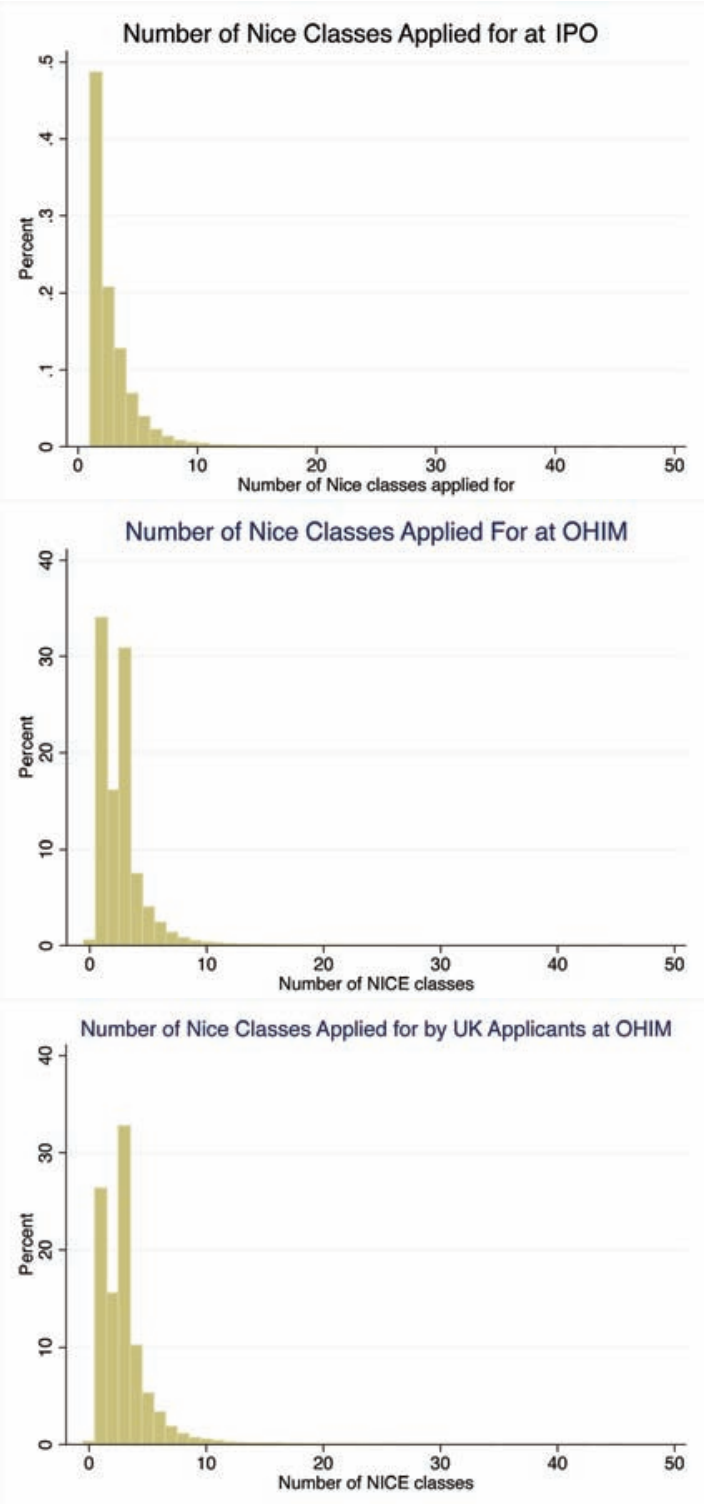

Note: The histogram for UK applications is based on 208,037 UK trade mark applications by UK based businesses made between 2000 and 2008. The histograms for OHIM applications are based on 851,989 and 91,231 trade mark applications made at OHIM between 1996 and 2010 by all applicants and UK based businesses respectively.

Figure 8 provides histograms of the frequency with which applicants choose to protect their marks in a specific number of classes. The left graph shows the behaviour of applicants at IPO where registration in each additional class induces further costs. As one might expect the largest number of applications is for protection in just one class. The frequency of registration in additional classes diminishes as additional classes are added. In contrast, the middle and right graphs show that applicants at OHIM (be they from the UK or not) very frequently choose to protect their trade marks in exactly three Nice classes. In the absence of the potential cost in terms of encouraging opposition we would expect no applicant at OHIM to pick fewer than three classes. The choice of one or two classes is still observed sufficiently frequently to suggest that opposition induces some firms to choose only one or two classes.

Now we turn to time trends in the numbers of classes which firms protect on their trade marks. We focus particularly on the proportion of marks 
protected in exactly three classes at IPO and OHIM and contrast this with less than three and more than three. The left graph in Figure 9 shows that at IPO the majority of applicants choose to register in one or two Nice classes. Also the graph shows that the proportion of applicants choosing less than/more than or exactly three classes is quite stable over time. In contrast the graphs (centre and right) depicting applicants' choices at OHIM show that over time the proportion of applications registered in three or more Nice classes is strongly increasing. In the case of UK applicants we can observe that the proportion of OHIM trade marks registered in exactly three classes was as high in 2010 as the proportion of marks registered in fewer than three Nice classes.

\section{Figure 9: Proportion of applications made in exactly (less /more than) three Nice}

\section{classes}
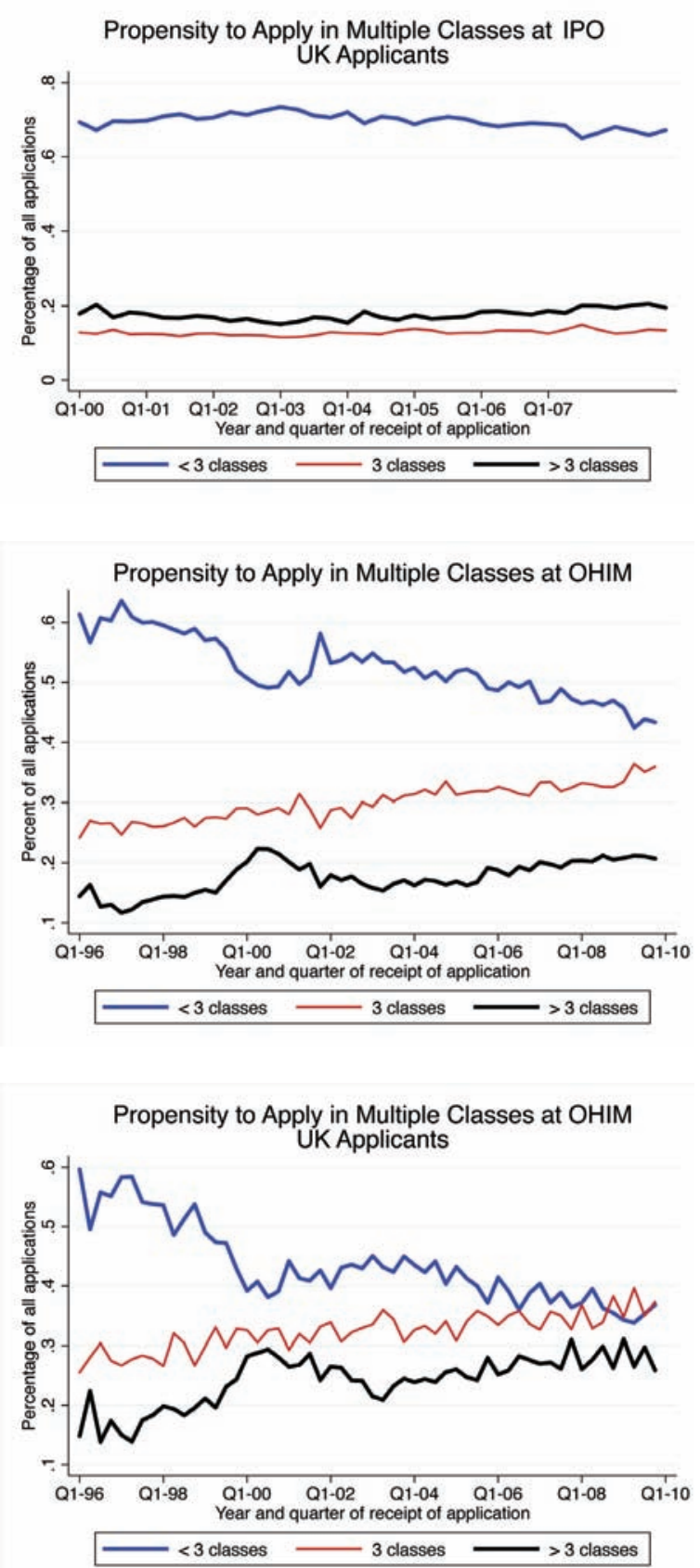

There are two explanations for the observed trends in the data from OHIM. The first is that firms are increasingly responsive to OHIM's pricing regime. The second is that there is a trend towards broader use of trade marks, i.e. that firms really are operating in markets related to several Nice classes more frequently. This second explanation seems less likely to be correct given the evidence from IPO data. We provide a brief analysis of opposition data to examine this question in more detail below.

Using data on trade mark opposition at OHIM before 2004 Table 2 above provides information on the probability that an opposition case ends in a particular way. Most interesting are outcomes that suggest a trade mark was overly broad to begin with. These are the outcomes Limited and Rejected in part. Table 2 shows that the probability that marks were limited or partly rejected in opposition procedures at OHIM was significantly higher for trade mark applications covering 2 or 3 Nice classes than for trade mark applications covering 1 or more than 3 classes - these differences are statistically significant at the $1 \%$ level. This can be explained if firms that do not have legitimate business in two or three Nice classes are being lured into applying for protection in additional classes by OHIM's pricing policy. In opposition procedures the excess classes are then stripped off leading to limitation or partial rejection of the mark. 
Table 2: Opposition Status by Number of Classes

\begin{tabular}{|c|c|c|c|c|c|}
\hline \multirow[t]{2}{*}{ Status } & & $1 \&>3$ & $2 \& 3$ & Classes & Tota \\
\hline & $\mathrm{N}$ & $\%$ & $\mathrm{~N}$ & $\%$ & $N$ \\
\hline Limited & 9,912 & 23.31 & 9,150 & 25.78 & 19,062 \\
\hline Rejected & 1,444 & 3.40 & 723 & 2.04 & 2,167 \\
\hline Rejected in part & 1,230 & 2.89 & 1,197 & 3.37 & 2,427 \\
\hline TM withdrawn & 8,065 & 18.96 & 6,531 & 18.40 & 14,596 \\
\hline Not duly entered - final & 58 & 0.14 & 57 & 0.16 & 115 \\
\hline Opposition rejected & 3,957 & 9.30 & 3,114 & 8.77 & 7,071 \\
\hline Opposition withdrawn & 7,387 & 17.37 & 5,554 & 15.65 & 12,941 \\
\hline No decision & 379 & 0.89 & 344 & 0.97 & 723 \\
\hline Admissible & 443 & 1.04 & 472 & 1.33 & 915 \\
\hline Agreement & 132 & 0.31 & 105 & 0.30 & 237 \\
\hline Commencement & 5,436 & 12.78 & 4,681 & 13.19 & 10,117 \\
\hline Cooling off & 4,082 & 9.60 & 3,565 & 10.04 & 7,647 \\
\hline Opposition filled & 1 & 0.00 & 0 & 0.00 & 1 \\
\hline Total & 42,526 & 100.00 & 35,493 & 100.00 & 78,019 \\
\hline
\end{tabular}

Source: OHIM Opposition data before 2004.

\subsubsection{A Policy Shock: Abolishing Refusals based on Relative Grounds Examination}

This section exploits the abolition of refusals based on relative grounds examination (RGE) at IPO in October of 2007 to investigate the importance of relative grounds examination as a hurdle for registration of trade marks. We find that indeed the removal of this hurdle had a concomitant effect on opposition rates at IPO and at OHIM. As we do not have data on the importance of relative grounds examination in preventing registration at IPO before October 2007 we cannot yet determine what percentage of trade mark applications is now registered that would previously have failed.

The analysis here relies on the comparison of the fate of trade mark applications at OHIM and IPO coming from UK applicants with the fate of trade mark applications at OHIM and IPO coming from non UK applicants. We seek to identify any differences in opposition rates for trade marks coming from UK applicants before and after October 2007. Under certain assumptions such differences will be solely due to the abolition of refusals on the basis of relative grounds examination. We use the level of trade mark opposition to trade marks from countries other than the UK to control for any other influences on trade mark opposition. This form of analysis is called differences-in-differences regression.

We find that after October 2007, when RGE was changed to a notification system in the UK, oppositions against trade marks sponsored by UK applicants at IPO increased relative to oppositions against non UK applicants. Simultaneously, oppositions against trade marks sponsored by UK applicants at OHIM seem to have decreased relative to oppositions against non UK applicants. 
This result would be commensurate with UK applicants using OHIM to circumvent IPO's RGE if they felt it might prevent them from registering trade marks. It is interesting to note that Table 3 below also demonstrates that opposition against UK trade marks at OHIM was more probable than opposition against the average trade mark at OHIM or against trade marks originating with German applicants at OHIM. This is to be expected if RGE has the effect of deflecting more problematic applications towards OHIM. However, the table also demon-strates that UK applicants faced higher opposition rates in the United Kingdom than foreign applicants. Finally, it should be noted that the analysis is based on relatively few periods of observation at IPO after the policy change. It may be that with longer time series the findings are less or more pronounced than those we describe below.

Background A trade mark application at any European trade mark office will not be registered if there are absolute grounds for the rejection of the mark. Absolute grounds for rejection are mostly harmonised at EU level. For instance functional characteristics of a good are usually not registrable as a trade mark as they do not serve to differentiate the versions of that good produced by one firm from those produced by other firms.

\begin{tabular}{|c|c|c|c|c|c|}
\hline Year & IPO & UK @ IPO & OHIM & UK@ OHIM & DE @ OHIM \\
\hline 1996 & & & .16555 & .18424 & .14904 \\
\hline 1997 & & & .17096 & .18683 & .18948 \\
\hline 1998 & & & 18762 & .21092 & 20911 \\
\hline 1999 & & & .18701 & .19619 & .20419 \\
\hline 2000 & .00056 & .00065 & .17640 & .1843 & .19054 \\
\hline 2001 & .00162 & .00176 & .16917 & .17950 & .18723 \\
\hline 2002 & .00331 & .00424 & .16372 & .17774 & .17276 \\
\hline 2003 & .01500 & .02017 & .15806 & .16431 & .15800 \\
\hline 2004 & .02663 & .03204 & .16482 & .17491 & .15800 \\
\hline 2005 & .02640 & .03045 & .15849 & .18178 & .17793 \\
\hline 2006 & .03002 & .03547 & 15018 & .1775 & .16925 \\
\hline 2007 & .04197 & .04794 & .13919 & .16034 & .16236 \\
\hline 2008 & .04195 & .04838 & .13281 & .15112 & .14869 \\
\hline Total & .02057 & .02556 & .15890 & .17683 & .17155 \\
\hline
\end{tabular}

Note: Opposition procedures at OHIM can take up to four years. As the trade mark application process at OHIM can also take up to two years and the data from OHIM were obtained in January 2010 the opposition rates for 2008 and 2009 in columns 3, 4 and 5 of the above table are likely to be too low. 
A number of trade mark offices also add the hurdle of relative grounds for the rejection of trade mark applications. In this case the office itself evaluates whether a trade mark application is so similar to that of an existing trade mark belonging to a different firm that it can create confusion on the part of the consumers. In this case the office will reject the application without involving the owner of the already registered trade mark. Finally, most offices also allow firms owning trade marks to oppose trade mark applications of other firms once the office has declared that it would otherwise register the marks. Opposition thus presents the last administrative hurdle before a trade mark is entered onto the trade mark register.

Until October of 2007 trade marks registered at IPO were examined both on absolute grounds and on relative grounds. In contrast, trade marks registered at OHIM were not examined on relative grounds. This means that effectively registration at OHIM was more easily obtained for trade marks than at IPO. On the other hand opposition assumes a more important role at OHIM in ensuring that trade marks are not registered that infringe on trade marks already registered there or elsewhere in Europe.

Table 3 above shows that opposition rates at OHIM are 10 times higher than those at IPO in 2003. However there seems to be a trend to increasing levels of opposition at IPO between 2003 and $2007 .{ }^{33}$

Analysis - Here we present results from differences in differences regressions based on data from OHIM and IPO. This analysis relies on two assumptions:

a) Any underlying trends in the data are common to applications coming from the UK and all other countries.

b) Applications of UK applicants are more strongly affected by the abolition of refusals based on RGE than applications of firms from outside the UK.

The second assumption is the more important one here. It is justified if non-UK applicants are less likely than UK applicants to seek to circumvent RGE by applying to OHIM for a trade mark. This seems a justifiable assumption here as a non-UK applicant who is applying to IPO rather than OHIM must have strong reasons to want just a UK trade mark. These reasons, on average will be stronger than those of UK applicants, for whom IPO is the default office, if they do not intend to trade outside the United Kingdom.

To see whether the abolition of refusals based on RGE had any significant effects on oppositions to trade marks of UK applicants we estimate the probability of opposition using OLS regressions (linear probability models). In each case the regression equation takes the following form:

$$
\operatorname{Pr}(\text { Opposition })=\alpha+\beta_{U K} D_{U K}+\beta_{07} D_{07}+\beta_{U K 07} D_{U K 07}+\gamma^{\prime} X+\epsilon \quad .
$$

The dependent variable is a dummy variable (coded 0 or 1 ) which is set to 1 if there was an opposition against a given application. If there were several oppositions against the same application these were recorded as separate observations in the data.

33 The data for IPO for the period before 2003 seem suspiciously low. We have not yet been able to determine what might be the reason for this. 
The main explanatory variables in the above regression consist of three dummy variables

$D_{U K}$ This variable is set to 1 if the applicant comes from the UK.

$D_{07}$ This variable is set to 1 from the fourth quarter of 2007 onwards.

$D_{U K 07}$ This variable is an interaction between the preceding two. It is the main variable of interest: it shows us whether the change in the probability of opposition for trade marks of UK applicants due to the policy change in October of 2007 was significantly different from the change in the probability of opposition for trade marks of applicants from other countries.

If this is the case we infer that the abolition of refusals based on RGE affected the behaviour of UK applicants.

When running the regressions we also control for a number of covariates that are subsumed under $X$ in the equation above. The precise list of covariates depends on the data set we used.

Opposition at OHIM - First, we discuss the regressions we ran for trade mark opposition at OHIM.

In case of the OHIM data we use ten additional covariates. One covariate captures aspects of firm strategy:

- New combination dummy: A measure of a change in strategy is a dummy variable that is set to one if the combination of Nice classes the firm is citing on its applications is new to that firm.

Three covariates measure firm characteristics for a subset of firms:

- Age: To proxy the age of the applying firm, we extract the age of the oldest trade mark in the portfolio of each applicant. This age measure is available where firms have cited previously existing trade marks (seniorities) in their applications for an OHIM trade mark.

- Seniorities: This variable contains a count of trade marks with seniorities which the firm applies for over the entire sample period. This provides an approximation to the size of the firm before 1996 if the firm cites previously existing trade marks in its applications for OHIM trade marks.

- No seniorities dummy: This dummy variable is set to one for firms that never rely on seniorities when applying to OHIM.

Two covariates capture differences between industries:

- Opposition rate This variable measures the proportion of previous applications opposed that are applying to a particular combination of Nice classes.

- Registration rate This variable measures the proportion of previous applications registered that are applying to a particular combination of Nice classes. 
The remaining covariates capture country, industry and time fixed effects:

- Country For each firm the country of origin is recorded. This information is included in the regressions as significant national differences between the trade marking strategies of firms can be detected. These are fixed over time.

- Nice dummies For each application the combination of Nice classes used is employed to capture industry specific differences in trade marking behaviour that are fixed over time.

- Quarter In aggregate time series of trade mark applications significant differences between the application rates in different quarters can be observed. Therefore we include dummy variables for quarters in the regressions below.

- Year Year dummies are included in most regressions below to capture effects of the business cycle or other shocks that are specific to individual years and affect all applicants equally.

\begin{tabular}{|l|llll|}
\multicolumn{5}{|c|}{ Table 4: Linear Probability Models of Opposition Rates at OHIM } \\
\hline \multirow{2}{*}{} & Base & DiD & $\begin{array}{l}\text { DiD \& Quarter } \\
\text { dummies }\end{array}$ & $\begin{array}{l}\text { DiD \& Time } \\
\text { Trend }\end{array}$ \\
\hline UK dummy & -0.005 & -0.003 & -0.003 & -0.003 \\
& $(0.006)$ & $(0.006)$ & $(0.006)$ & $(0.006)$ \\
2007 dummy & & $0.020^{* * *}$ & $-0.204^{* * *}$ & $-0.035^{* * *}$ \\
UK x 2007 & & $(0.004)$ & $(0.004)$ & $(0.002)$ \\
dummy & & $-0.009^{* *}$ & $-0.009^{*}$ & $-0.007^{*}$ \\
Time Trend & & $(0.003)$ & $(0.003)$ & $(0.003)$ \\
& & & & $-0.001^{* * *}$ \\
Constant & $0.244^{* * *}$ & & & $(0.000)$ \\
& $(0.014)$ & $\left(0.244^{* * *}\right.$ & $0.252^{* * *}$ & $0.404^{* * *}$ \\
\hline R-squared & 0.054 & 0.055 & $(0.014)$ & $(0.017)$ \\
N & 785547 & 785547 & 0.056 & 0.051 \\
\hline
\end{tabular}

$\dagger p<0.10,{ }^{*} p<0.05,{ }^{* *} p<0.01,{ }^{* * *} p<0.001$. Robust standard errors in parentheses, standard errors clustered at firm level. Models include country, year and Nice class dummies and additional covariates. Results from Differences in Differences Models Estimated by OLS.

Table 4 provides results of running the differences in differences model (Equation (2)) using OHIM opposition data. We only provide coefficients for the most important variables here. The table contains four different regressions. The first column contains results from a regression including only the covariates. This is a baseline against which to judge the remaining regressions. The next three columns set out results from models in which we changed the way in which time fixed effects were modelled. These effects capture unobserved shocks that vary over time but affect all applications within a given year or quarter equally. The fourth column sets out results from a model in which we include a time trend - this is often done to test the robustness of results from differences in differences models. 
We find that adding the dummy variables for the policy change in October 2007 and the interaction $(U K \times 2007)$ to the baseline model has significant effects. More specifically we have a robust negative effect of the interaction dummy in all three models. This shows that at the time of the abolition of refusals based on RGE (October 2007) the probability of opposition for trade marks from UK applicants at OHIM decreased relative to the opposition probability for applicants from other countries at IPO.

We also find that there is no significant overall difference between the probability of opposition for UK applications and our control group (applications from Austria) in these regressions.

\section{Figure 10: Opposition Probabilities by Quarter}

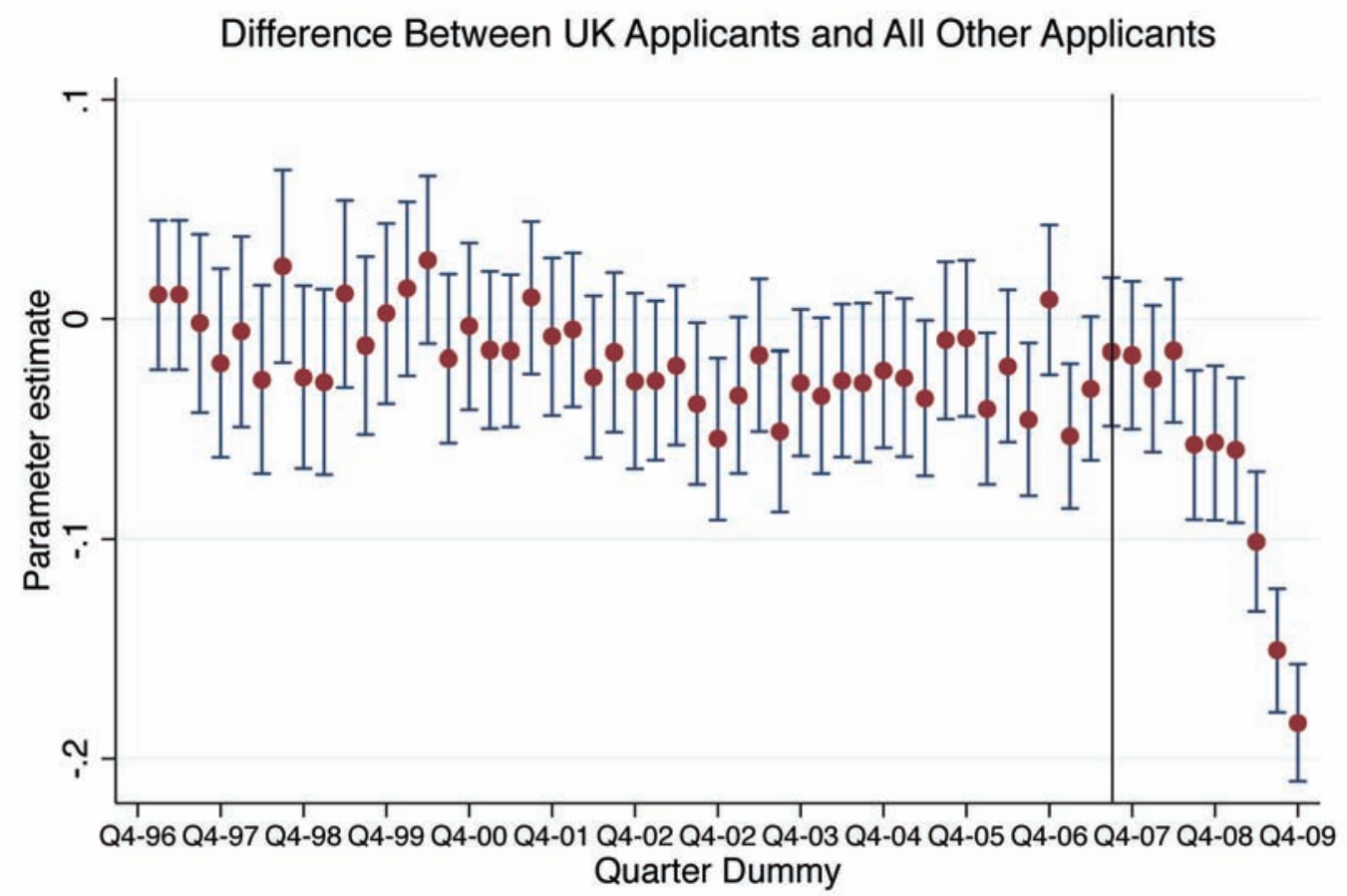

To further test this result we provide a graph from a model in which we interact the UK dummy with quarter dummies for each quarter in our data (Figure 10). This reveals that there does seem to be a strong effect for UK applicants from about a year after the policy change onwards. It is not clear how reliable this finding is as the process of application and opposition proceedings at OHIM can take up to 2 and 4 years respectively. However, unless trade mark applications from UK applicants are treated systematically differently from those of other applicants in this process this should not affect the results. Since effects seem to become very substantial as we near the end of the sample period it would be important to test these findings with updated information from OHIM.

Opposition at IPO - Here we discuss the regressions we ran with data on oppositions at IPO. As noted in the discussion of Table 3 it may be that the data used here is not entirely complete. 
Trade Mark Cluttering: An Exploratory Report Commissioned by IPO

The data we obtained from IPO did not contain as much information as the data we obtained from OHIM. In some cases this is not surprising as the notion of "seniority" is specific to OHIM. This means that we do not have information about the age of preceding trade marks which a UK mark may be based on. Nor do we have information on the size of the firm's trade mark portfolio. We did not have the time to construct covariates capturing industry specific opposition and registration rates from the UK data yet. Nor do we have more than the distinction between UK and non-UK applicants in our data at this time. This could be done in a follow up study to test the robustness of our findings here.

The covariates used in the regressions reported below capture industry and time fixed effects:

- Nice dummies For each application the combination of Nice classes used is employed to capture industry specific differences in trade marking behaviour that are fixed over time.

- Quarter In aggregate time series of trade mark applications significant differences between the application rates in different quarters can be observed. Therefore we include dummy variables for quarters in the regressions below.

- Year Year dummies are included in most regressions below to capture effects of the business cycle or other shocks that are specific to individual years and affect all applicants equally.

Table 5 provides results of running the differences in differences model (Equation (2) ) using IPO opposition data. As above we only provide coefficients for the most important variables. The table contains four different regressions constructed in analogous fashion to those presented in Table 4 above.

We find that adding the dummy variables for the policy change in October 2007 and the interaction ( UK $\times 2007$ ) to the baseline model has significant effects here too. More specifically we have a robust positive effect of the interaction dummy in all three models. This shows that at the time of the abolition of refusals based on RGE examination (October 2007) the probability of opposition for trade marks from UK applicants at OHIM increased relative to the opposition probability for applicants from other countries. 
Table 5: Linear Probability Models of Opposition Rates at IPO

\begin{tabular}{|l|llll|}
\hline & Base & DiD & DiD \& Quarter dummies & DiD \& Time Trend \\
\hline United Kingdom dummy & & $0.008^{* * *}$ & $0.008^{* * *}$ & $0.008^{* * *}$ \\
& & $(0.001)$ & $(0.001)$ & $(0.001)$ \\
2007 dummy & & $-0.009^{* * *}$ & $-0.017^{* * *}$ & $-0.009^{* * *}$ \\
UK x 2007 dummy & & $(0.002)$ & $(0.002)$ & $(0.001)$ \\
& & $0.009^{* * *}$ & $0.009^{* * *}$ & $0.0009^{* * *}$ \\
Time Trend & $(0.002)$ & $(0.002)$ & $(0.002)$ \\
& & & & $0.001^{* * *}$ \\
Constant & & & & $(0.000)$ \\
& -0.001 & $-0.007^{* *}$ & $0.038^{* * *}$ & $-0.240^{* * *}$ \\
\hline R-squared & $(0.002)$ & $(0.002)$ & $(0.002)$ & $(0.005)$ \\
N & 0.017 & 0.018 & 0.018 & 0.017 \\
\hline
\end{tabular}

$\dagger p<0.10,{ }^{*} p<0.05,{ }^{* *} p<0.01,{ }^{* * *} p<0.001$. Robust standard errors in parentheses, standard errors clustered at firm level. Models include country, year and Nice class dummies and additional covariates. Results from Differences in Differences Models Estimated by OLS.

As in the descriptive data (Table 3) we find that the baseline probability of opposition for UK applications at IPO is higher than that of applicants from other countries. This may be due to selection effects as we can expect applicants from other countries to be larger and better represented firms than the average UK applicant.

Summary of Results - Overall this analysis provides two findings. The policy change abolishing refusals based on RGE at IPO had the effect of reducing the probability that a UK applicant's trade mark was opposed at OHIM relative to applicants from other countries. At the same time the probability that a UK applicant's trade mark was opposed at IPO increased relative to applicants from other countries.

These effects could be explained if UK applicants used OHIM to circumvent RGE. If so, some of these attempts to register trade marks that RGE might have caught, then got held up in opposition proceedings at OHIM leading to a higher rate of opposition there. Once RGE was dropped at IPO it seems such trade marks were registered with IPO again. This explanation for the effects of removing RGE at IPO will need to be tested more thoroughly before it can be taken seriously. In particular UK applicants seeking to circumvent RGE would have had to contend with much higher opposition rates at OHIM. Whether this could have been attractive is unclear as we do not have data on the proportion of trade marks failing due to RGE at IPO in the relevant period.

One way of doing this would be to look more carefully at the outcomes of opposition cases before and after 2007, to use more recent data and to look more carefully at the types of applicants who might have been switching between offices. All three of these tests could be undertaken if more up to date and detailed data were employed. It would also be possible to try to quantify the effects of removing RGE on the composition of registered marks at both offices. 
Focusing more closely on cluttering it would also seem important to establish whether RGE had an effect on the breadth of trade mark applications. Did examiners exert an influence on applicants to stick to classes that they were likely to be able to prove use or was RGE not effectual in this regard?

\subsubsection{Many Simultaneous Applications}

In this section we discuss evidence on cluttering derived from the analysis of simultaneous applications at OHIM. The analysis is taken from ongoing work by Georg von Graevenitz.

Simultaneous applications arise especially frequently in the pharmaceuticals sector. This is because regulators of medical products (e.g. FDA, EMEA) are increasingly concerned also about the naming of such products. This is due to the increased danger for consumers arising from confusion of trade marks. Lallemand (2011) cites the example of drugs Losec and Lasix, the first is a proton pump inhibitor to treat heartburn whereas the second is used to treat hypertension. Due to the similarity in the pronunciation of both drugs, the US Food and Drug Administration (FDA) forced Losec's producer AstraZeneca to adopt a new name for Losec. AstraZeneca chose the name Prilosec, which was eventually confounded with Prozac by a pharmacist. The anecdote serves to illustrate the importance of brand names in the pharmaceutical industry.

In the pharmaceutical industry, therefore, cluttering will make it more difficult for firms to find an appropriate name as the search for existing marks becomes more costly and rejections by the different authorities becomes more likely. This increases uncertainty, in particular because there remains some degree of subjectivity in the decision process. As a result, pharmaceutical firms routinely apply for several trade marks for a single drug well before the drug has passed all regulatory hurdles.

It is not clear as yet whether the phenomenon of simultaneous applications is restricted to pharmaceutical applicants only. The analysis here reveals how firms react within the trade mark system when it becomes harder to ensure that a single trade mark is successfully registered due to actions of further regulators next to the trade mark office. We believe that there may be other firms facing similar problems of multiple regulatory hurdles for their trade marks. For instance firms applying to many trade mark offices simultaneously due to the international nature of their business. At present we lack the data resources to investigate this question further. We hope to rectify this in the near future.

For the purposes of our analysis here simultaneous applications are applications made by one firm on one day using the same set of Nice classes. Here we treat each such event as an application event which is intended to produce at least one trade mark for an underlying product. Then every time a firm applies for and successfully registers two or more trade marks simultaneously one or more applications are surplus.

This method of measurement may be misleading if firms seek to simultaneously register both a word mark, a figure and possibly a smell or a shape to mark a product. Therefore, we distinguish between these different types of applications and only count those cases in which a firm simultaneously applies for more than one of each type of trade mark. Seven types of trade mark are distinguished. ${ }^{34}$

34 There are Word marks, figurative marks, three dimensional marks, colour marks, olfactory marks, holograms and a residual category. 
Between 1.4.1996 and 1.1.2010 there are 598,224 application events in the OHIM data for which we have sufficient information to include them in the analysis. Of these $11.73 \%(70,182)$ are events in which a firm applied for more than one trade mark of one type simultaneously.

As discussed in the Section 2.1.3 simultaneous applications of trade marks are prevalent in the pharmaceutical industry. To simplify the analysis we analysed which Nice classes are frequently/ very rarely cited on trade mark applications that are also applied for in class 5 , the main class for pharmaceuticals. Using this information we divide the set of Nice classes into three sets. These are labelled pharmaceuticals, artifacts and food \& household. Table 6 below illustrates which Nice classes fall into these sets.

\begin{tabular}{|l|ccc|}
\multicolumn{4}{|c|}{ Table 6: Distribution of Trade Mark Applications } \\
\cline { 2 - 4 } & \multicolumn{3}{|c|}{ Type of Industry } \\
\cline { 2 - 4 } Nice Classes & Artifacts & Food \& Household & Pharmaceuticals \\
& $6,7,9,12,16,18$ & $11,20,21$, & $1,3,5$, \\
& $19,25,28,33,35$, & $29,30,31$, & $10,13,44$ \\
& $36,37,38,39,41$, & 32,42 & \\
& 43,45 & & Chemicals, Laundry, \\
Description & Metals, Machines, & Lighting, Furniture, & Pharmaceuticals, \\
& Scientific Apparatus & Household utensils & Medical Apparatus, \\
& Vehicles, Leather, & Food, Coffee, & Firearms, \\
& Building, Clothing, & Produce, Beer & Medical Services \\
\hline Games,,.. & & 76,201 \\
\hline
\end{tabular}

Using this segmentation of Nice classes we ascribe trade mark application events to one of these three groups plus a fourth group (pharma \& artifacts) where a mark cites both Nice classes highly related and unrelated to pharmaceuticals.

Next we build on this categorisation to analyse descriptively how the average number of trade marks per application event changes over time at OHIM.

Figure 11 sets out our findings. The figure shows clearly that simultaneous applications occur much more frequently in pharmaceuticals applications than in any of the other groups. Only towards the end of the sample period do we observe increases in simultaneous applications in the food \& household category which approach levels we otherwise only observe in pharmaceuticals. 


\section{Figure 11: Cluttering by Simultaneous Applications at OHIM}

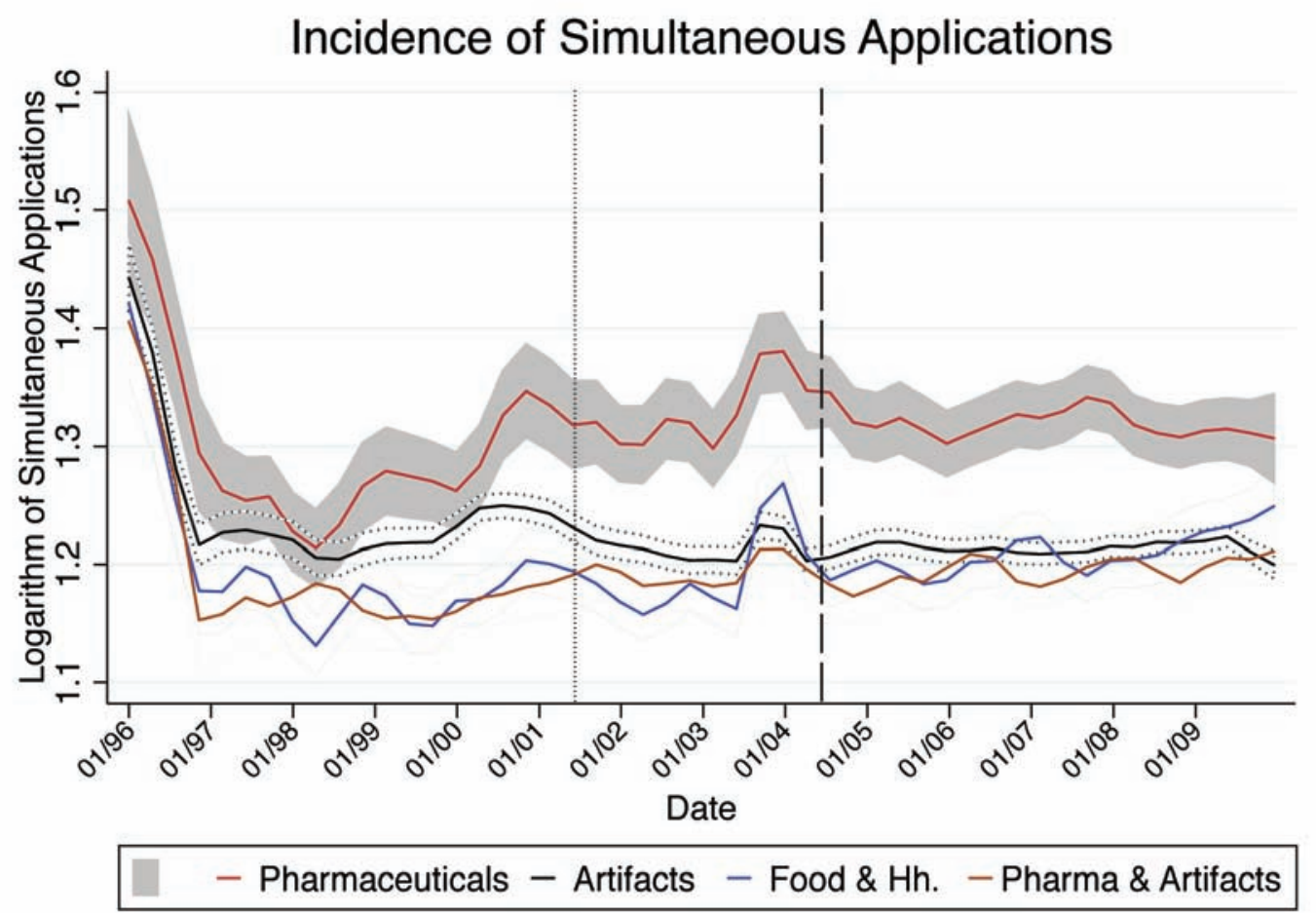

Figure 12 provides additional detail about the applications outside of pharmaceuticals. This reveals that in Food \& Household there is a significant increase in simultaneous applications at the end of the sample period. Additional data will be necessary to determine whether this was the beginning of a trend or just a short term event.

Turning to the significance of simultaneous applications we have investigated how large the impact of the phenomenon is on the trade mark register. If we assume that for every trade mark application event in which more than one mark was applied for all marks beyond the first mark were surplus, then simultaneous application alone added 124,751 surplus trade marks to the register at OHIM between 1997 and 2009. This would represent $15.44 \%$ of all trade mark applications in this period. This is likely to be an overestimate of the true number of excess marks. If we restrict the analysis just to those marks that are registered as pharmaceutical trade marks then 26,210 of 108,251 applications may be surplus, i.e. $24.21 \%$.

Additional evidence that simultaneous application events really add surplus trade marks to the register comes from an analysis of trade mark renewals. We can undertake this for all 84,136 OHIM registered trade marks with a registration before 1999. From this group we drop all trade mark applications for which firms did not renew a single concurrent trade mark leaving 58,359 applications. Next we drop all those cases in which firms only applied for a single mark $(38,497)$ and we are left with 19,862 registered marks. Out of these $14.79 \%$ of trade marks were not renewed. This percentage is surprisingly close to that obtained above. 


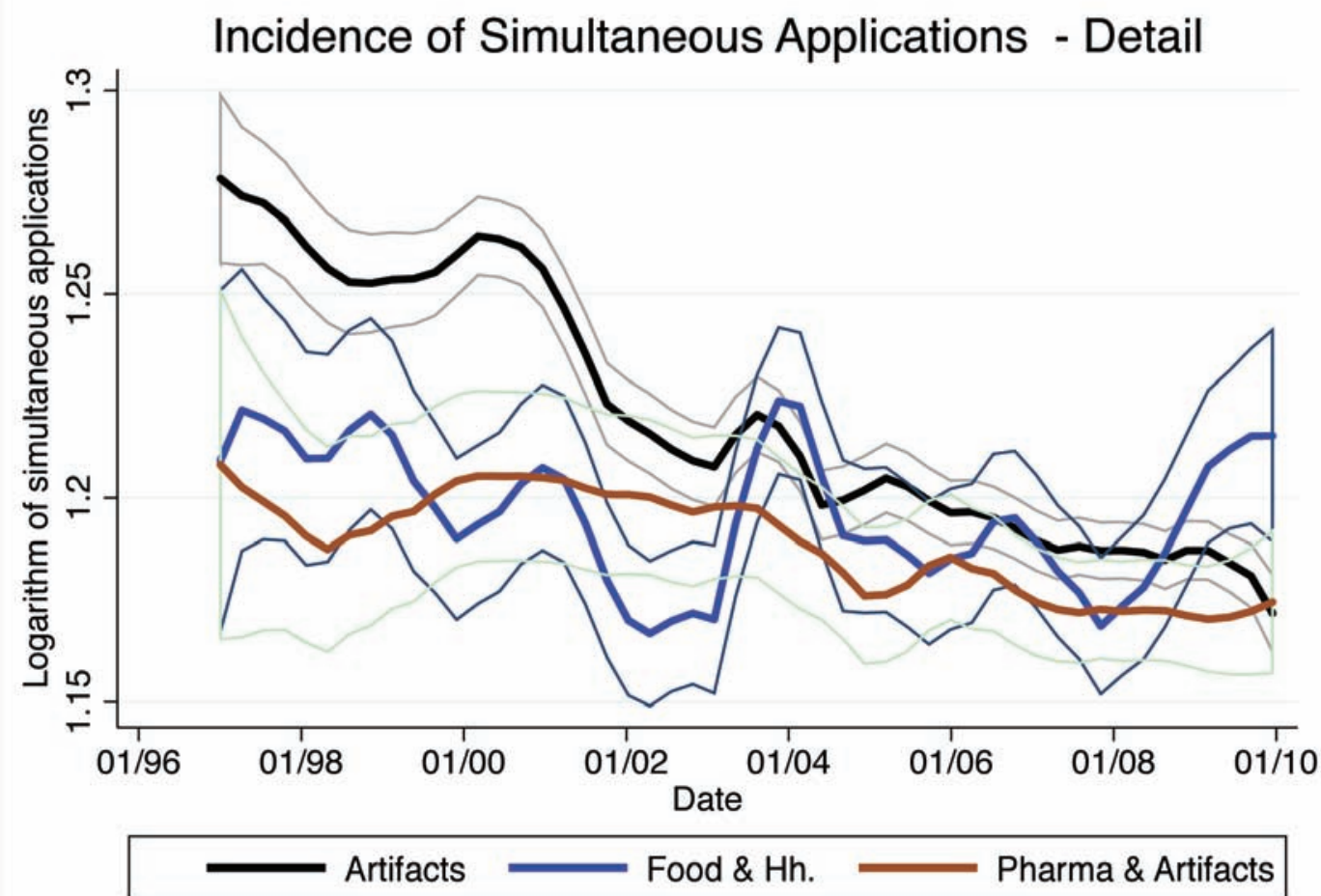

Further analysis of these types of applications will be necessary to better identify which trade marks really are registered unnecessarily. For instance, it may be that in some cases firms are registering trade marks simultaneously because they are building a brand family. However, in these cases we would expect to see them renew all parts of this family. Additionally, we would be able to identify marks within the same brand family on the basis of their similarity. We will investigate this in future work.

In spite of these caveats, it is clear that the proportion of registered trade marks that are surplus due to unnecessary simultaneous applications is likely to be high. This is clear from expert interviews and discussions with firm representatives we have had in the recent past. This conclusion is also supported by our analysis of trade mark renewals cited here.

At present we are sure only that simultaneous applications arise in the area of pharmaceuticals. As we show above even in this case they still make up a non negligible number of unused trade marks. We expect that there are additional industries and classes of firms (e.g. those applying across several jurisdictions at the same time) that may also resort to the strategy of simultaneous applications. Analysis of this question will require creation of more comprehensive data-bases. 


\section{Conclusion}

This report explores the phenomenon of "cluttering" of trade mark registers. We provide a conceptual discussion of "cluttering," review the existing literature, and analyse data on trade mark applications and oppositions at IPO and OHIM for indications of cluttering.

Cluttering occurs when the existence of a dense set of similar names in a field raises trade mark costs for other firms or even inhibits market entry of new competitors. In searching for metrics we take the view that cluttering is likely to arise where firms hold trade marks that are overly broad or unused, raising search costs for later applicants seeking marks in the same product class.

The report distinguishes between three mechanisms that can lead to cluttering:

- Firms may apply for more classes than are needed for their present use. (This may be encouraged by the OHIM 3 for 1 pricing policy.)

- Firms may have to apply for several marks for one product where there is an industry regulator, as in pharmaceuticals, which may reject some names that could lead to confusion across products and jeopardise the health and safety of patients.

- Firms may have a genuine anticipation of broadening their product range and wish to register names that are a logical extension of their existing naming scheme.

We have also considered a series of mechanisms that work against cluttering:

- The costs of registration generally rise if application is made in a larger number of classes.

- Where an application is opposed on the basis of an earlier mark that has been registered for over five years, if proof of use of the latter is requested and cannot be demonstrated, the opposition will fail.

- Any unused mark will eventually lapse if its owner chooses not to renew.

- Opportunities exist for licensing or sale of the registered mark if the two products are in non-competing markets.

The existing literature considering the nature and existence of trade mark cluttering reviewed in this report is very sparse and typically produced by legal scholars, not by those working in economics or business disciplines. There is thus no precedent for empirical economic analysis of this topic and this study breaks new ground. We present descriptive and inferential statistics on trade mark activity by UK firms using the UK and EU routes to trade mark registration. We also analyse data on opposition activity within both jurisdictions.

The evidence provided in the report in Section 3.1 shows trade marks are frequently registered in several classes by all firms. Even so, larger firms file on average in more classes than smaller firms. Across economic sectors there is little variation in the number of classes filed through time, with just three exceptions of utilities, communications and real estate, each of which fluctuates. 
In Section 3.2.1 drawing on research from Europe, we report direct evidence that applicants perceive cluttering to be a problem in specific fields and countries.

Section 3.2.2 explores whether the 3-for-1 pricing policy used at OHIM encourages more applications in multiple classes. The analysis of opposition rates suggests this is the case as instanced both by trends in filing and by the higher probability of opposition for marks registered in 3 classes.

Section 3.2.3 shows some evidence that suggests firms seek to avoid mechanisms such as relative grounds examination which can prevent cluttering.

We also show in Section 3.2.4 that firms in specific fields increasingly resort to multiple simultaneous applications to ensure that they will obtain at least one trade mark.

Clearly further work is needed to determine whether cluttering has already become a systemic problem for the trade mark systems that is comparable to the effect of patent thickets for patent systems. The findings of this study suggest this is a topic worth pursuing. Even where we learn that cluttering is not a problem, it will be worthwhile clarifying which rules (e.g. use requirements) within the trade mark system prevent this from happening. Such understanding would help trade mark offices to prevent cluttering from arising due to inadvertent abolition or watering down of these important rules. 


\section{References}

BESSEN, J. and M. J. MEURER (2008): Patent Failure, Princeton, N.J.: Princeton University Press.

BOLDRIN, M. and D. LEVINE (2008): Against Intellectual Monopoly, Cambridge, U.K.: Cambridge University Press.

CABRAL, L. M. B. (2000): Introduction to Industrial Organization, Cambridge, Mass.: MIT Press.

COUNCIL REGULATION (EC) NO. 40/94 (1994): "European Commission," Official Journal, L $11,1-49$.

DEPO, B. (2010): "Same Procedure Every Year: Locus Standi as an Effective Barrier to Trade Mark Revocation for Non-use in Poland," Journal of Intellectual Property Law \& Practice, 5.

ECONOMIDES, N. S. (1988): "The Economics of Trade marks," Trade mark Reporter, 78, 523 -539 .

FEDERAL TRADE COMMISSION (2003): "To Promote Innovation - The Proper Balance of Competition and Patent Law and Policy," Report, GPO.

GREENHALGH, C., Rogers. M., Schautschick. P., and Sena. V. (2011): "Trade Mark Incentives," Tech. rep., Intellectual Property Office.

GRIFFITHS, A. (2007): "Trade Marks Plus? The Role of Trade Marks in the Global Economy and the Danger of Overprotection," Liverpool Law Review, 28, 107-141.

(2009): "The Organisational Role of Trade Marks," Mimeo.

GRINDLEY, P. C. and D. J. TEECE (1997): "Managing Intellectual Capital: Licensing and CrossLicensing in Semiconductors and Electronics," California Management Review, 39, 8-41.

HALL, B., C. HELMERS, M. ROGERS, and V. SENA (2011): "The Importance of Patents and Other Formal Intellectual Property in Comparison to Informal Protection Methods," Report, IPO.

HALL, B. H. and R. ZIEDONIS (2001): "The Patent Paradox Revisited: An Empirical Study of Patenting in the U.S. Semiconductor Industry, 1979-1995," Rand Journal of Economics, 32, $101-128$.

HARGREAVES, I. (2011): "Digital Opportunity: A Review of Intellectual Property and Growth," Report, IPO.

HELMERS, C. and M. ROGERS (2009): "Trade Marks and Performance in UK firms," in Trade Marks, Brands and Competitiveness, ed. by T. da Silva Lopes and P. Duguid, New York, N.Y.: Routledge.

HELMERS, C., M. ROGERS, and P. SCHAUTSCHICK (2011): "Intellectual Property at the Firm-Level in the UK: The Oxford Firm-Level Intellectual Property Database," Oxford University, Department of Economics Discussion Paper, 546.

IYAMA, S. (2005): “The USPTO's Proposal of a Biological Research Tool Patent Pool Doesn't Hold Water," Stanford Law Review, 57, 1223-1241. 
JAFFE, A. B. and J. LERNER (2004): Innovation and Its Discontents: How Our Broken Patent System is Endangering Innovation and Progress, and What to Do About It., Princeton, N.J.: Princeton University Press.

KUR, A., R. HILTY, and R. KNAAK (2011): "Study on the Overall Functioning of the European Trade Mark System," Tech. rep., Max Planck Institute for Intellectual Property and Competition Law Munich.

LALLEMAND, J. (2011): "Pharmaceutical Trade marks: how to Survive the Name Game," Tech. rep., Thomson Compumark.

LANDES, W. M. and R. A. POSNER (1987): "Trade mark Law: An Economic Perspective," Journal of Law and Economics, 30, 265-309.

LEI, Z. and B. D. WRIGHT (2009): "Why Weak Patents? Rational Ignorance or Pro-'customer' Tilt?" Tech. rep.

LEMLEY, M. A. and C. SHAPIRO (2005): "Probabilistic Patents," Journal of Economic Perspectives, 19, 7598.

NATIONAL RESEARCH COUNCIL (2004): Patent System for the 21st Century, Report of the Board on Science, Technology, and Economic Policy, Washington, D.C.: National Academies Press.

ROB, R. and A. FISHMAN (2005): "Is Bigger Better? Customer Base Expansion Through Wordof-mouth Reputation," Journal of Political Economy, 113, 1146-1162.

ROGERS, M., C. HELMERS, and C. GREENHALGH (2009): "An Analysis of the Characteristics of Small and Medium Enterprises that use Intellectual Property," Report, IPO.

SCHULTE, D. (1995a): "The Madrid Trade mark Agreement's Basis in Registration-Based Systems: Does the Protocol Overcome Past Biases (Part I)," J. Pat. \& Trade mark Off. Soc'y, $77,595$.

(1995b): "The Madrid Trade mark Agreement's Basis in Registration-Based Systems: Does the Protocol Overcome Past Biases (Part II)," J. Pat. \& Trade mark Off. Soc'y, 77, 729.

SHAPIRO, C. (2001): "Navigating the Patent Thicket: Cross-Licenses, Patent Pools, and Standard-Setting," in Innovation Policy and the Economy, ed. by A. Jaffe, J. Lerner, and S. Stern, NBER.

SUTTON, J. (2007): "Market Structure: Theory and Evidence," Handbook of industrial organization, 3, 2301-2368.

VON GRAEVENITZ, G., S. WAGNER, K. HOISL, B. HALL, D. HARHOFF, P. GIURI, and A. GAMBARDELLA (2007): The Strategic Use of Patents and its Implications for Enterprise and Competition Policies, Report for the European Commission.

WINNER, E. (1982): “Trade mark Registration - What's the Use?" Idea, 23, 49. 

Trade Mark Cluttering: An Exploratory Report Commissioned by IPO

\section{Appendix}

\section{Nice classification}

\begin{tabular}{|c|c|}
\hline Class Number & Description \\
\hline \multicolumn{2}{|l|}{ Goods Classes } \\
\hline 1 & Chemicals \\
\hline 2 & Paints, varnishes, and lacquers \\
\hline 3 & Substances for laundry use \\
\hline 4 & Industrial oils and grease \\
\hline 5 & Pharmaceutical and sanitary preparations \\
\hline 6 & Common metals \\
\hline 7 & Machines and machine tools \\
\hline 8 & Hand tools and implements \\
\hline 9 & Scientific apparatus \\
\hline 10 & Medical apparatus \\
\hline 11 & Lighting and heating \\
\hline 12 & Vehicles \\
\hline 13 & Firearms \\
\hline 14 & Precious metals and jewellery \\
\hline 15 & Musical instruments \\
\hline 16 & Paper, packaging and printing \\
\hline 17 & Rubber and gum \\
\hline 18 & Leather \\
\hline 19 & Building materials \\
\hline 20 & Furniture \\
\hline 21 & Household or kitchen utensils \\
\hline 22 & Ropes, sails, and bags \\
\hline 23 & Yarns and threads for textile use \\
\hline 24 & Textiles and textile good \\
\hline 25 & Clothing, footwear \\
\hline 26 & Lace, pins, and needles \\
\hline 27 & Materials for covering floors \\
\hline 28 & Games, toys, and decoration \\
\hline 29 & Meat, fish, and vegetable \\
\hline 30 & Coffee, bread, and salt \\
\hline 31 & Agricultural and forestry \\
\hline 32 & Beers \\
\hline 33 & Alcoholic beverages \\
\hline 34 & 34 Tobacco, matches \\
\hline \multicolumn{2}{|l|}{ Service Classes } \\
\hline 35 & Advertising and business \\
\hline 36 & Insurance and financial services \\
\hline 37 & Building and construction \\
\hline 38 & Telecommunications \\
\hline 39 & Transport \\
\hline 40 & Treatment of materials \\
\hline 41 & Education, sport, and culture \\
\hline 42 & Other services \\
\hline 42 & Scientific, technological \\
\hline 43 & Services for providing food and drink \\
\hline 44 & Medical services \\
\hline 45 & Personal and social services \\
\hline
\end{tabular}


Trade Mark Cluttering: An Exploratory Report Commissioned by IPO

Figures relating to Section 3.1

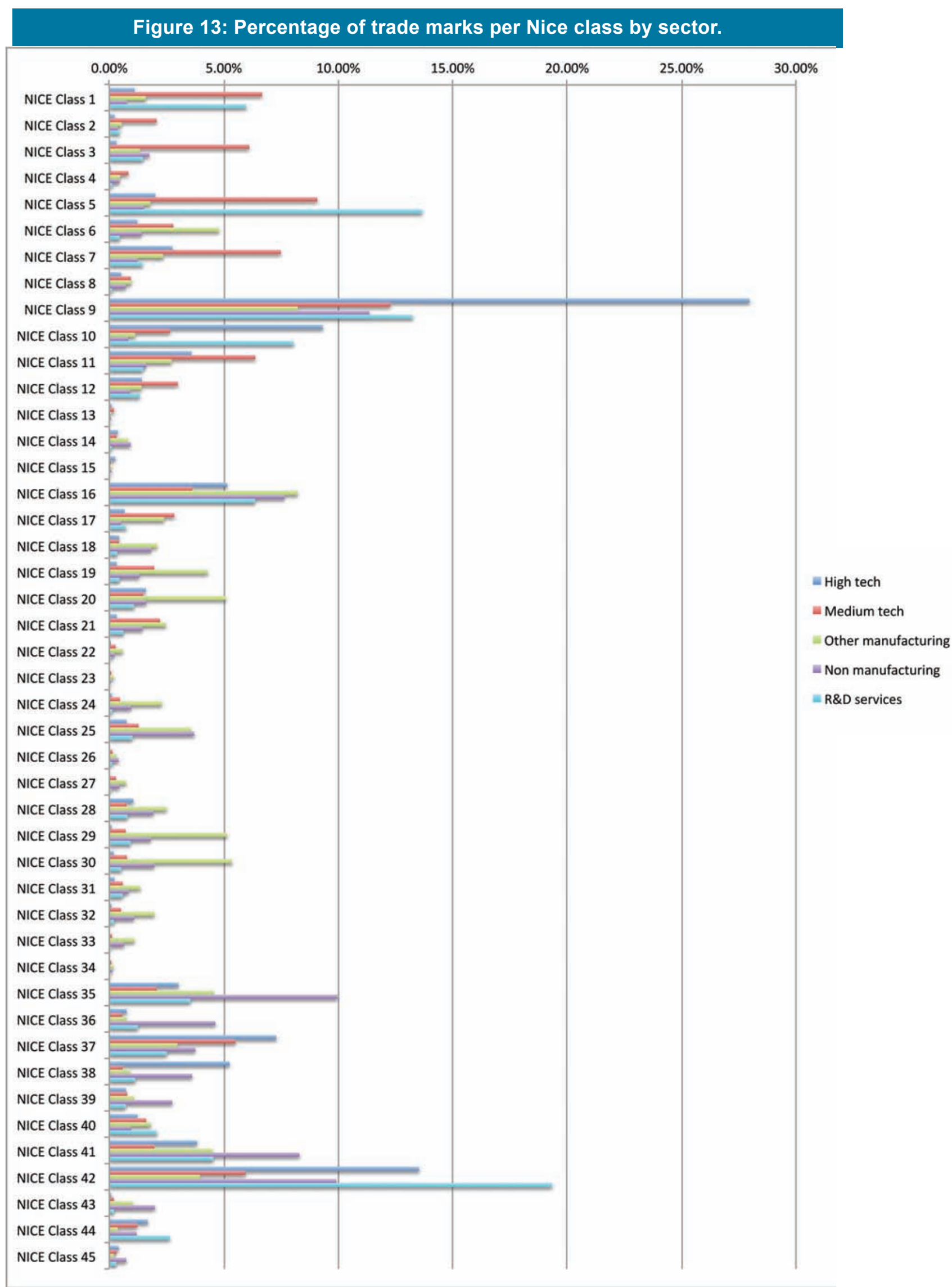




\section{Figure 14: Percentage of trade marks per Nice class by firm size.}

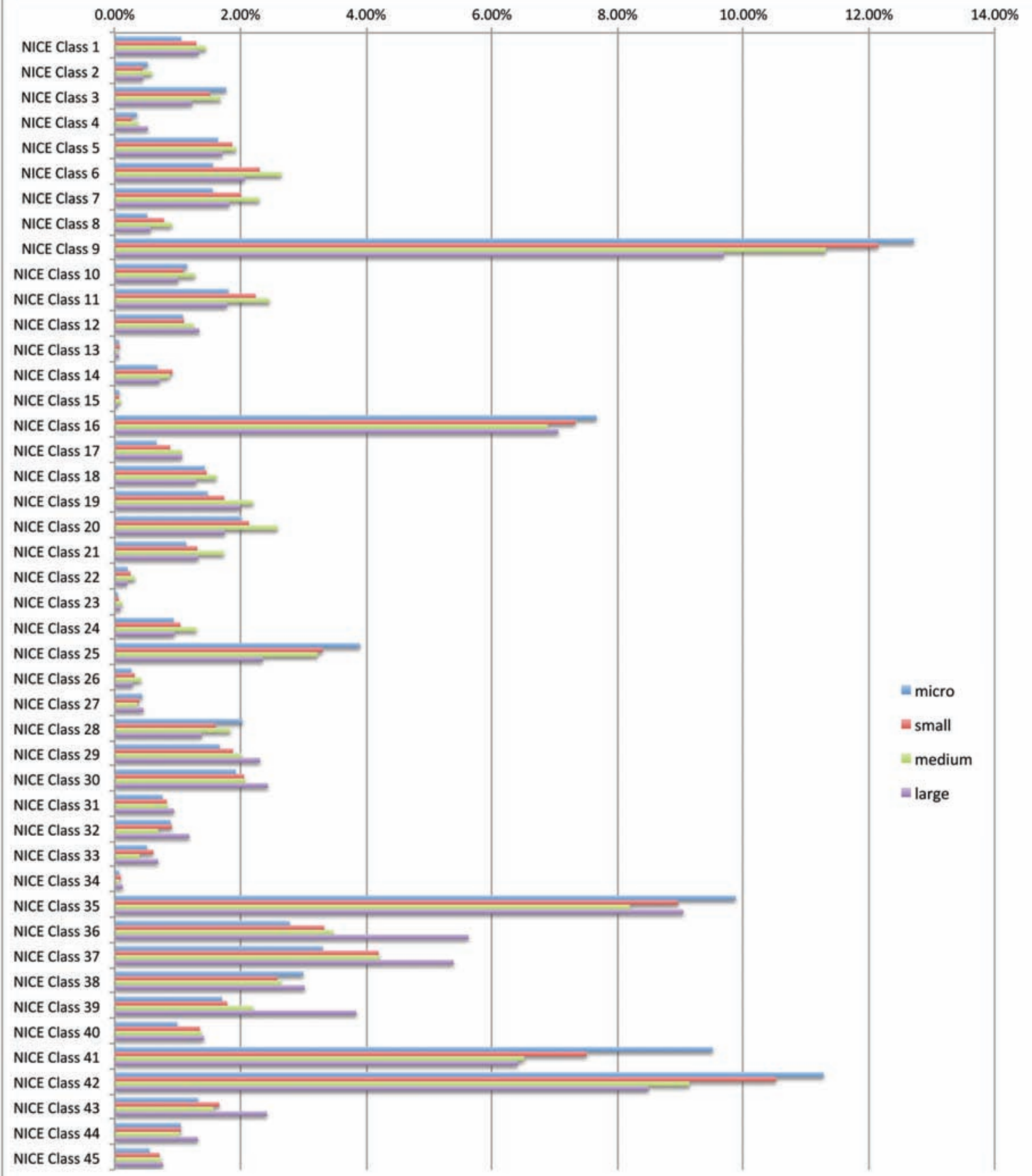


Trade Mark Cluttering: An Exploratory Report Commissioned by IPO

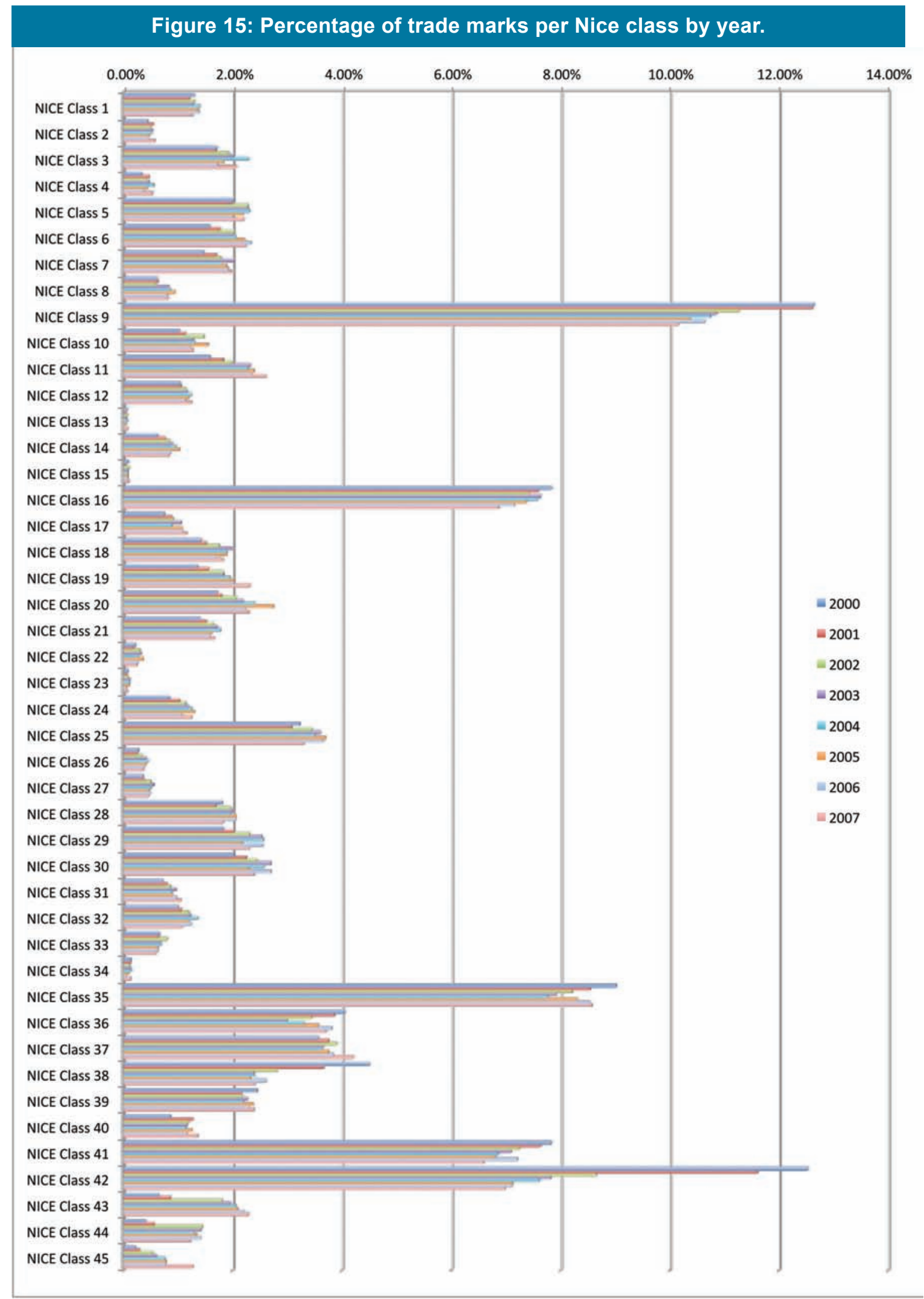





Concept House

Cardiff Road

Newport

NP10 8QQ

Tel: 03003002000

Minicom: 03000200015

Fax: 01633817777

For copies in alternative formats please contact our Information Centre.

When you no longer need this booklet, please recycle it.

ISBN: 978-1-908908-29-2 